\title{
Plane Curves Associated to Character Varieties of 3-Manifolds
}

\author{
D. Cooper, M. Culler ${ }^{1}$, H. Gillet ${ }^{1}$, D. D. Long ${ }^{1}$ and P. B. Shalen ${ }^{2}$
}

\section{$\S 1$. Introduction.}

Consider a compact 3-manifold $M$ with boundary consisting of a single torus. The papers [CS1], [CS2] and [CGLS] discuss the variety of characters of $\mathrm{SL}_{2}(\mathbf{C})$ representations of $\pi_{1}(M)$, and some of the ways in which the topological structure of $M$ is reflected in the algebraic geometry of the character variety. We will describe in this paper a certain affine algebraic curve $D_{M}$ which is naturally associated to the character variety of $M$. A basis $B=\{\mathrm{L}, \mathrm{M}\}$ for the peripheral subgroup of $M$ determines an embedding $p_{B}$ of $D_{M}$ into $\mathbf{C}^{*} \times \mathbf{C}^{*}$ with coordinates $l$ and $\mathrm{m}$. The closure in $\mathbf{C}^{2}$ of $p_{B}\left(D_{M}\right)$ is a plane algebraic curve and therefore is defined by a polynomial $A_{M, B}(\mathrm{l}, \mathrm{m})$ that, after certain normalizations, is uniquely determined up to multiplication by constants. The polynomial $A_{M, B}$ is effectively computable and is an invariant of the manifold $M$ together with the choice of basis $B$. The results in this paper describe how geometric properties of the character variety, and hence topological properties of $M$, are reflected by the polynomial $A_{M, B}$.

In the case that $M$ is the complement of a knot $K$ in a homology 3 -sphere we may take the basis $B$ to consist of the longitude and meridian of $K$. With the usual orientation conventions this basis is well-defined modulo the involution which inverts both the longitude and meridian. It will follow from the construction of $D_{M}$ that if the basis $B^{\prime}$ is obtained from $B$ by inverting both generators then the regular maps $p_{B}$ and $p_{B^{\prime}}$ have the same image. Thus $A_{K}:=A_{M, B}=A_{M, B^{\prime}}$ is an invariant of the knot.

The polynomial $A_{M, B}$ displays, in a striking way, information about the incompressible surfaces in $M$. This involves the Newton polygon of $A_{M, B}$, which is the convex hull of the integer lattice points in the plane whose coordinates arise as degrees of monomials in $A_{M, B}$. Using the main result of [CS1] we show (Theorem 3.4) that "boundary slopes are boundary slopes," that is that the slope of each side of the Newton polygon of $A_{M, B}$ equals the boundary slope of an incompressible surface in $M$ which is associated to an

\footnotetext{
${ }^{1}$ Partially supported by the Sloan Foundation and the National Science Foundation.

${ }^{2}$ Partially supported by the National Science Foundation.
} 
action of $\pi_{1}(M)$ on the affine building of $\mathrm{SL}_{2}(F)$ for a certain function field $F$. To say that an incompressible surface $S$ is associated to the action means that $S$ is produced by a transversality construction using a $\pi_{1}(M)$-equivariant map from the universal cover of $M$ to the building, which is a tree in this case. Such a surface is not unique, but has a well-defined boundary slope. We will consider surfaces which have the minimal number of boundary components among all incompressible surfaces associated to the action. These are called reduced surfaces.

To describe the proof of Theorem 3.4, we consider the following projective embedding of $D_{M}$. Embed $\mathbf{C}^{*} \times \mathbf{C}^{*}$ in $\mathbf{P}^{2}$ as the set $\{(\mathrm{l}: \mathbf{m}: z) \mid \mathbf{l} \neq 0, \mathbf{m} \neq 0, z=1\}$. Let $\overline{D_{M}}$ denote the closure of the image of $D_{M}$ in $\mathbf{P}^{2}$. By a point at infinity of $\overline{D_{M}}$ we mean a point lying on one of the three axes $l=0, \mathrm{~m}=0$ or $z=0$. It follows from the construction of $D_{M}$ that each local branch of $\overline{D_{M}}$ through a point at infinity determines an ideal point of a curve of $\mathrm{SL}_{2}(\mathbf{C})$ characters of $\pi_{1}(M)$. The technique of [CS1], which constructs an incompressible surface for each ideal point of a curve of characters, extends to provide an incompressible surface for each local branch of $\overline{D_{M}}$ through a point at infinity. Moreover, each local branch determines a discrete valuation $v$ on the function field of the component of $\overline{D_{M}}$ containing it. The boundary slope of the incompressible surface associated to the branch can be expressed in terms of $v$ as $-v(\mathrm{l}) / v(\mathrm{~m})$.

Theorem 3.4 is thus a consequence of the following statement. Given coprime integers $p$ and $q$ there exists a local branch of $\overline{D_{M}}$ through a point at infinity, for which the associated valuation $v$ satisfies $v(\mathrm{l})=p$ and $v(\mathrm{~m})=q$, if and only if the Newton polygon of $A_{M, B}$ has a side of slope $-p / q$. The link between the valuation and the Newton polygon is provided by the notion of a Puiseaux parametrization of a local branch of a curve. Each side of the Newton polygon determines a Puiseaux parametrization of some local branch of $\overline{D_{M}}$ through a point at infinity. This parametrization provides a local coordinate with respect to which a function on the local branch can be expanded as a Laurent series. The valuation assigns to a function the degree of the leading term of its Laurent series.

We construct our Puiseaux parametrization of a local branch of $\overline{D_{M}}$ so that $\mathrm{m}$ is a power of the local coordinate. It then turns out that the the leading coefficient of the Laurent series that represents $l$ carries topological information about the reduced surface associated to the local branch. This coefficient can be described in terms of the valuation. In general, if $f$ and $g$ are elements of a field $F$ with a discrete rank 1 valuation $v$, and if $s$ is the greatest common divisor of $v(f)$ and $v(g)$, then the element $g^{v(f) / s} f^{-v(g) / s}$ determines a well-defined element of the residue field. We will denote this element by $\tau_{v}(f, g)$. When $v$ is the valuation determined by a local branch of $\overline{D_{M}}$ through a point at infinity we have $\tau_{v}(\mathrm{l}, \mathrm{m})=a_{0}^{v(\mathrm{l})}$. We show (Theorem 5.7) that $\tau_{v}(\mathrm{l}, \mathrm{m})$ is always a root of unity and that the order of $\tau_{v}(\mathrm{l}, \mathrm{m})$ divides the number of boundary components of each component of $S$. As corollary we have that the leading coefficient $a_{0}$ is a root of unity. 
In fact, we give two different proofs that $\tau_{v}(\mathrm{l}, \mathrm{m})$ is a root of unity with order dividing the number of boundary components of $S$. Both proofs begin by producing a representation of $\pi_{1}(M)$ into $\mathrm{SL}_{2}(F)$, where $F$ is a finite extension of the function field of $Y \subset D_{M}$ equipped with a discrete valuation $v^{\prime}$ which is an extension of $v$. One argument involves the algebraic K-theory of the field $F$, while the other uses the action of $\pi_{1}(M)$ on the affine building of $\mathrm{SL}_{2}(F)$ determined by the representation. There are intriguing parallels between these two arguments which presumably are due to the existence of a deeper connection between K-theory and the theory of affine buildings over function fields.

In the $\mathrm{K}$-theoretic argument the functions $\mathrm{l}$ and $\mathrm{m}$, regarded as elements of $F$, are eigenvalues of the images of $\mathrm{L}$ and $\mathrm{M}$ on a common eigenspace. These determine an element $\{l, \mathfrak{m}\}$ of $K_{2}(F)$. The square of $\{l, m\}$ is, in a sense, the K-theoretic fundamental class of the peripheral torus of $M$ and hence vanishes. The result follows from the existence of a homomorphism (the tame symbol) from $K_{2}(F)$ to $C^{*}$ which sends $\{x, y\}$ to $\tau_{v^{\prime}}(x, y)^{n}$ for a certain integer $n$. It is immediate from the definition that $\tau_{v}(x, y)=\tau_{v^{\prime}}(x, y)$. It follows from the construction of $S$ that the exponent $n$ divides the number of boundary components of $S$.

The tree-theoretic argument also involves eigenvalues and homological considerations, but in quite a different setting. If an element $A$ of $\mathrm{SL}_{2}(F)$ fixes a vertex $w$ of the affine building then $A$ is conjugate to an element $A^{\prime}$ of $\mathrm{SL}_{2}(\mathcal{O})$, where $O$ denotes the valuation ring in $F$. The conjugacy determines an identification of the link of the vertex $w$ with with the complex projective line so that the induced action of $\mathrm{SL}_{2}(\mathcal{O})$ factors through the standard action of $\mathrm{SL}_{2}(\mathbf{C})$. If an edge $e$ with initial vertex $w$ is fixed by $A$ then it is identified with an eigenspace of the reduction of $A^{\prime}$ modulo the maximal ideal. The associated complex eigenvalue is called the eigenvalue of $A$ associated to the fixed (directed) edge $e$.

If $S$ is an incompressible surface associated to the action of $\pi_{1}(M)$ on the building of $\mathrm{SL}_{2}(F)$ then the subgroup $\pi_{1}(S)$ is contained in the stabilizer of some edge $e$. Thus each element of $\pi_{1}(S)$ has an eigenvalue associated to the edge $e$. The boundary components of a component of $S$ can be regarded as elements of $\pi_{1}(S)$ whose product is contained in the commutator subgroup of the stabilizer of $e$, and hence has eigenvalue 1. For any boundary component the eigenvalue associated to $e$ is either $\tau_{v}(\mathrm{l}, \mathrm{m})$ or $\tau_{v}(\mathrm{l}, \mathrm{m})^{-1}$. A topological argument with a homological flavor shows that if $S$ is reduced then, for all of the boundary components of a component of $S$, the eigenvalues associated to $e$ are equal. This implies that $\tau_{v}(\mathrm{l}, \mathrm{m})$ is a root of unity with order dividing the number of boundary components of each component of $S$. (So the tree-theoretic argument gives a slightly stronger result than does the K-theoretic argument.)

The paper is organized as follows. The definitions of $D_{M}$ and $A_{M, B}$ are given in $\S 2$. In $\S 3$ we explain the connection between Puiseaux parametrizations and valuations and prove that boundary slopes are boundary slopes. The K-theoretic arguments are given in 
$\S 4$ and the tree-theoretic arguments in $\S 5$. In $\S 6$ we discuss the relationship between $A_{K}$ and the Alexander polynomial of $K$, where $K$ is a knot in a homology sphere. Finally, in $\S 7$ we give computations of $A_{K}$ for several knots $K$.

This paper is as much an account of overlapping independent efforts by its many authors as it is a collaboration. Many of the ideas presented here were also discovered by Andrew Casson.

\section{$\S 2$. Definitions.}

In this section we give the definition of the curve $D_{M}$ and the polynomial $A_{M, B}$. The 3 -manifold $M$ is assumed to be compact, oriented and to have a single torus boundary component. We will assume that a basis $B=\{\mathrm{L}, \mathrm{M}\}$ of the free abelian group $\pi_{1}(\partial M)$ has been fixed, and in the case where $M$ is a knot complement in a homology 3-sphere that $L$ and $M$ are the longitude and meridian respectively.

2.1. We will follow the notational conventions of [CS1], [CS2] and [CGLS]. Given a finitely generated group $\pi$ we denote by $R(\pi)$ the variety of representations of $\pi$ in $\mathrm{SL}_{2}(\mathbf{C})$. The variety of characters of $\mathrm{SL}_{2}(\mathbf{C})$ representations of $\pi$ is denoted $X(\pi)$ and $t: R(\pi) \rightarrow X(\pi)$ is the canonical projection which, by [CS1], is surjective. If $\gamma$ is an element of $\pi$ then $I_{\gamma}$ will be the function defined by $I_{\gamma}(\chi)=\chi(\gamma)$, and may be regarded as an element of the function field of any irreducible component of $X(\pi)$. When $\pi$ is the fundamental group of a manifold $N$ we may write $R(N)$ and $X(N)$ for the representation and character varieties of $\pi$. To construct the curve $D_{M}$, where $M$ is a compact 3-manifold with boundary a torus, we use the restriction map $r: X(M) \rightarrow X(\partial M)$ induced by the inclusion of $\pi_{1}(\partial M)$ into $\pi_{1}(M)$.

2.2. The curve $D_{M}$. Let $\Delta \subset R(\partial M)$ be the subvariety consisting of diagonal representations. There is an isomorphism $p_{B}$ from the affine variety $\Delta$ to $\mathbf{C}^{*} \times \mathbf{C}^{*}$ defined as follows: if $\rho$ is the representation defined by

$$
\rho(\mathrm{L})=\left[\begin{array}{cc}
l & 0 \\
0 & \mathrm{l}^{-1}
\end{array}\right] \text { and } \rho(\mathrm{M})=\left[\begin{array}{cc}
\mathrm{m} & 0 \\
0 & \mathrm{~m}^{-1}
\end{array}\right]
$$

then $p_{B}(\rho)=(\mathrm{l}, \mathrm{m})$. It is easily verified that $t$ restricts to a surjection $t_{\Delta}: \Delta \rightarrow X(\partial M)$, and that $t_{\Delta}$ is a finite map which is generically 2-to-1. In fact $X(\partial M)$ may be identified with the quotient of $p_{B}(\Delta)$ under the involution $\sigma$ which interchanges $l$ with $l^{-1}$ and $\mathrm{m}$ with $\mathrm{m}^{-1}$; the map $t_{\Delta}$ is the composition of $p_{B}$ with the quotient map.

Denote by $X^{\prime}(M)$ the union of the irreducible components $Y^{\prime}$ of $X(M)$ such that the closure of $r\left(Y^{\prime}\right)$ is 1-dimensional. For each component $Z^{\prime}$ of $X^{\prime}(M)$ let $Z$ be the curve 
$\left.t_{\Delta}^{-1} \overline{\left(r\left(Y^{\prime}\right)\right.}\right) \subset \Delta$. We define $D_{M}$ to be the union of the curves $Z$ as $Z^{\prime}$ varies over all components of $X^{\prime}(M)$.

Note that if $B^{\prime}$ is the basis consisting of $\mathrm{L}^{-1}$ and $\mathrm{M}^{-1}$ then $p_{B^{\prime}}=\left(\mathrm{l}^{-1}, \mathrm{~m}^{-1}\right)=p_{B} \circ \sigma$. Thus $p_{B}$ and $p_{B^{\prime}}$ have the same image. Having made this observation, it will no longer be necessary to indicate dependence upon the (fixed) basis $B$ of $\pi_{1}(M)$, so the subscript $B$ will be suppressed. We will identify $D_{M}$ with its image in $\mathbf{C}^{*} \times \mathbf{C}^{*}$. If $Y$ is an irreducible component of $D_{M}$ we will write $\bar{Y}$ for the projective completion of $Y$ in $\mathbf{C} P^{2}$ and $\tilde{Y}$ for the smooth projective model of $Y$. By an ideal point of $D_{M}$ we will mean a point of $\tilde{Y}$, for some irreducible component $Y$ of $D_{M}$, which corresponds to a point of $\bar{Y}-Y$. There are finitely many ideal points of $D_{M}$, each of which determines a discrete rank one valuation on the function field $\mathbf{C}(Y)$ for some irreducible component $Y$ of $D_{M}$. If $v$ is a valuation on $\mathbf{C}(Y)$ which corresponds to an ideal point of $D_{M}$ then at least one of $l, m, l^{-1}$ or $\mathrm{m}^{-1}$ is not contained in the valuation ring of $v$.

Proposition. Let $Y$ be an irreducible component of $D_{M}$. Then there exists a finite extension $F$ of $\mathbf{C}(Y)$ and a representation $P: \pi_{1}(M) \rightarrow \mathrm{SL}_{2}(F)$ such that

$$
P(\mathrm{~L})=\left[\begin{array}{cc}
l & 0 \\
0 & \mathrm{l}^{-1}
\end{array}\right] \text { and } P(\mathrm{M})=\left[\begin{array}{cc}
\mathrm{m} & 0 \\
0 & \mathrm{~m}^{-1}
\end{array}\right],
$$

where $\mathrm{l}$ and $\mathrm{m}$ are regarded as elements of the coordinate ring of $Y$.

ProOf. By the construction of $D_{M}$ there is an irreducible component $Y^{\prime}$ of $X(M)$ and a curve $Y_{\partial M}$ contained in $X(\partial M)$ such that the restriction of $r$ to $Y^{\prime}$ is a dominating map to $Y_{\partial M}$. Also the restriction of $t_{\Delta}$ to the component $Y$ is a dominating map to $Y_{\partial M}$. Finally, since $t: R(M) \rightarrow X(M)$ is surjective we may choose a curve $Y_{R} \subset R(M)$ such that the restriction of $r \circ t$ to $Y_{R}$ is dominating. Thus the function fields of $Y_{R}$ and $Y$ are both finite extensions of that of $Y_{\partial M}$. Moreover, since $Y_{R}$ is a curve in $R(M)$ there is a tautological representation $P_{1}: \pi_{1}(M) \rightarrow \mathrm{SL}_{2}\left(\mathbf{C}\left(\mathrm{Y}_{\mathrm{R}}\right)\right)$. Let $F$ be a common finite extension of $\mathbf{C}(Y)$ and $\mathbf{C}\left(Y_{R}\right)$. We may regard $P_{1}$ as a representation of $\pi_{1}(M)$ into $\mathrm{SL}_{2}(\mathrm{~F})$. Since $F$ contains $l$ and $m$, which are the eigenvalues of the commuting matrices $P_{1}(\mathrm{~L})$ and $P_{1}(M)$, the representation $P_{1}$ is conjugate in $G L_{2}(F)$ to a representation $P$ satisfying the conclusion of the proposition.

2.3. The polynomial $A_{M}$. By a defining polynomial of a plane curve we mean a polynomial which vanishes exactly on the curve and has no repeated irreducible factors. Such a polynomial is unique up to multiplication by non-zero constants. We define $A_{M}(\mathrm{l}, \mathrm{m})$ to be the defining polynomial of the closure of $D_{M}$ in $\mathbf{C} \times \mathbf{C}$. 
Proposition. After multiplication by a non-zero constant the coefficients of $A_{M}$ can be taken to be integers.

Proof. It suffices to show that the curve $X^{\prime}(M)$ is defined by polynomial equations with integral coefficients. For this we use a theorem attributed to Weil [La, Ch. III Theorem 7]. This theorem asserts that an ideal $I$ in a polynomial ring $K\left[x_{1}, \ldots, x_{n}\right], K$ a field, has a minimal field of definition $k \subset K$. Moreover an automorphism of $K$ leaves $I$ invariant if and only if it fixes $k$. Thus we need only show that $X^{\prime}(M)$ is invariant under any automorphism of the field of complex numbers (i.e under the the diagonal action of the automorphism on affine space.) It is clear that the variety $R(M)$ is defined over $\mathbf{Q}$. Since the projection to $X(M)$ is a polynomial map with integral coefficients, the variety $X(M)$ is also defined over $\mathbf{Q}$. Similarly, the restriction map $r: X(M) \rightarrow X(\partial M)$ is a polynomial map with integral coefficients, so $r(X(M))$ is defined over $\mathbf{Q}$. If a variety defined over $\mathbf{Q}$ is invariant under an automorphism of the complex numbers then the set of generic points on the variety is also invariant; it follows that the automorphism must take components of the variety to components of the same dimension. Therefore, since $X^{\prime}(M)$ is defined to be the union of the components of $X(M)$ whose image under the restriction map is 1-dimensional, $X^{\prime}(M)$ must be invariant under any automorphism of the complex numbers.

2.4. It is very often (but not always) the case that every component of $X(M)$ has dimension 1 , so that we actually have $X^{\prime}(M)=X(M)$. In particular we have the following.

Proposition. There are no 0-dimensional components of $X(M)$. Moreover, if $M$ contains no closed incompressible surface then every component of $X(M)$ has dimension 1.

Proof. It is shown in [CS1] that the characters of reducible representations form a closed algebraic subset of $X(M)$, and that any component of $X(M)$ which contains the character of an irreducible representation has dimension at least 1 . Thus a 0 -dimensional component of $X(M)$ would have to be a character of a reducible representation. But it is also shown in [CS1] that any reducible character in $X(M)$ is the character of a diagonal representation. It follows that a component of $X(M)$ which consists entirely of characters of reducible representations must have dimension greater than or equal to the first Betti number of $M$. Therefore $X(M)$ can have no 0-dimensional component.

If there is a component of $X(M)$ with dimension greater than 1 then for any peripheral element $\gamma$ in $\pi_{1}(M)$ there is a curve contained in $X(M)$ on which the function $I_{\gamma}$ restricts to a constant function. By the main theorem of [CS1] this implies that either $M$ contains a closed incompressible surface or $\pm[\gamma]$ is a boundary slope of an incompressible surface in $M$ for every peripheral element $\gamma$. The second alternative would contradict a 
theorem of Hatcher $[\mathrm{H}]$ which states that there are only finitely many boundary slopes of incompressible surfaces in $M$.

2.5. The subvariety $X^{\prime}(M)$ always has at least one component of reducible characters since any homomorphism $h: \pi_{1}(M) \rightarrow \mathbf{Z}$ gives rise to a subvariety of $X^{\prime}(M)$ consisting of characters of representations which factor through $h$. In the case where $M$ is a knot complement in a homology 3 -sphere, if $Y$ is a component of $X^{\prime}(M)$ consisting of reducible characters then the curve $\tilde{Y}$ in $\Delta$ is just the line $l=1$. (This is because the longitude is mapped to the identity by any reducible representation of $\pi_{1}(M)$.) Thus $l-1$ is a factor of $A_{K}(\mathrm{l}, \mathrm{m})$ for any knot $K$ in a homology sphere, and if $K$ is the trivial knot then $A_{K}(l, \mathrm{~m})=\mathrm{l}-1$.

2.6. Proposition. If $K$ is a hyperbolic knot then $A_{K}(\mathrm{l}, \mathrm{m}) \neq \mathrm{l}-1$.

ProOF. It follows from [CS2] that if $Y^{\prime}$ is an irreducible component of $X(M)$ which contains the character of a discrete faithful representation then $Y^{\prime}$ is 1-dimensional and the function $I_{\gamma}$ is non-constant on $Y^{\prime}$ for any non-trivial peripheral element $\gamma$. This implies that the closure $Y$ of $r\left(Y^{\prime}\right)$ is an irreducible component of $X(\partial M)$, of dimension at most 1 , which admits a non-constant function. Thus $Y$ is 1-dimensional and hence determines a component of $D_{M}$. Since the trace function $I_{\mathrm{L}}$ is non-constant on $Y^{\prime}$, it follows that the component of $D_{M}$ corresponding to $Y$ is different from the line $l=1$.

2.7. Proposition. If $K$ is a non-trivial $(p, q)$-torus knot then $A_{K}(\mathrm{l}, \mathrm{m})$ is divisible by $\mathrm{lm}^{p q}+1$. In particular, $A_{K}(\mathrm{l}, \mathrm{m}) \neq \mathrm{l}-1$.

Proof. The fundamental group of the complement $M$ of the $(p, q)$-torus knot has presentation $\left\langle x, y: x^{p}=y^{q}\right\rangle$. If $m p+n q=1$ then we may take $\mathrm{M}=x^{n} y^{m}$ and $\mathrm{L}=$ $x^{p} M^{-p q}$. A family of irreducible representations of $\pi_{1}(M)$ can be constructed by sending $x$ and $y$ to non-commuting elements of $\mathrm{SL}_{2}(\mathbf{C})$ of order $2 p$ and $2 q$ respectively. After conjugation, such a representation can be taken to restrict to a diagonal representation of $\pi_{1}(\partial M)$, which is then contained in $D_{M}$. The relation $\mathrm{L}=x^{p} \mathrm{M}^{-p q}$ immediately implies that the closure of the set of diagonal representations obtained this way is the curve $\operatorname{lm}^{p q}=-1$. Thus $\operatorname{lm}^{p q}+1$ is a factor of $A_{K}(l, m)$.

2.8. A point of intersection of $D_{M}$ with one of the lines $m= \pm 1$ corresponds to a representation $\rho: \pi_{1}(M) \rightarrow \mathrm{SL}_{2}(\mathbf{C})$ for which $\rho(M)$ has trace \pm 2 . Since $\rho(M)$ and $\rho(\mathrm{L})$ commute we either have $\rho(\mathrm{M})= \pm I$ or $\operatorname{tr} \rho(\mathrm{L})= \pm 2$. If $M$ is a knot complement in $S^{3}$ 
then $\pi_{1}(M)$ is generated by conjugates of $M$. Therefore in this case $\rho(M)= \pm 1$ implies that $\rho$ has abelian image and hence that $\rho(\mathrm{L})=I$. This shows that if $K$ is a knot in $S^{3}$ then there are non-negative integers $k_{1}$ and $k_{2}$ such that $A_{K}(l, \pm 1)=(l-1)^{k_{1}}(l+1)^{k_{2}}$.

The intersection points of the line $\mathrm{l}=1$ with the other components of $D_{M}$ are related to the Alexander polynomial; see $\S 6$.

2.9. Proposition. If $K$ is knot in a homology sphere then $A(\mathrm{l}, \mathrm{m})$ involves only even powers of $\mathrm{m}$.

Proof. Identify the cyclic group $\mathbf{Z}_{2}$ with the subgroup $\pm 1 \subset \mathbf{C}^{*}$. Any homomorphism $\alpha: \pi_{1}(M) \rightarrow \mathbf{Z}_{2}$ determines an involution of $R(M)$ which sends a representation $\rho$ to the representation $\rho_{\alpha}$ defined by $\rho_{\alpha}(g)=\alpha(g) \rho(g)$. In the case where $M$ is a knot complement in a homology 3-sphere the unique non-trivial homomorphism from $\pi_{1}(M)$ to $\mathbf{Z}_{2}$ sends $\mathbf{M}$ to -1 . The corresponding involution of $R(M)$ induces an involution of $D_{M}$ which sends $(l, \mathfrak{m})$ to $(l,-\mathrm{m})$. Thus $A_{K}$ is invariant under this involution, giving the result.

\section{$\S 3$. Boundary slopes and the Newton polygon}

One of the important aspects of the relationship between the algebraic geometry of the character variety and the topological structure of $M$ is described by the main theorem of [CS1] which associates incompressible surfaces in $M$ to ideal points of curves in $X(M)$. In this section we reinterpret this theorem in terms of the curve $D_{M}$ and show that boundary slopes of incompressible surfaces are displayed by the coefficients of $A_{M}$; the slope of each side of the Newton polygon of $A_{M}$ is equal to the boundary slope of an incompressible surface associated to an ideal point of $D_{M}$.

3.1. Recall that a slope is an unoriented homotopy class of non-trivial simple closed curves on $\partial M$; that is a pair $\{[ \pm \gamma]\}$ of primitive homology classes in $H_{1}(\partial M$; Z). If $S$ is an incompressible surface with non-empty boundary in $M$ then $\partial S$ is a family of parallel simple closed curves on $\partial M$ and hence determines a slope, called the boundary slope of $S$. If, as in our situation, a basis of $H_{1}(\partial M ; \mathbf{Z})$ is given then a slope can be represented by an element of $\mathbf{Q} \cup\{\infty\}$ in the usual way. In our case the slope $\{ \pm(a \mathrm{~L}+b \mathrm{M})\}$ is represented by the rational number $b / a$. 
Proposition. To each ideal point $x$ of $D_{M}$ there corresponds an incompressible surface with non-empty boundary in $M$. If $v$ is the valuation on $\mathbf{C}\left(D_{M}\right)$ associated to $x$ then the boundary slope of this incompressible surface is $-v(\mathrm{l}) / v(\mathrm{~m})$.

Proof. The construction of the incompressible surface proceeds exactly as in [CS1]. Let $Y$ be the irreducible component of $D_{M}$ and $v$ the valuation on $\mathbf{C}(Y)$ associated to $x$. By Proposition 2.2 there is a representation $P$ of $\pi_{1}(M)$ into $\mathrm{SL}_{2}(F)$ where $F$ is a finite extension of $\mathbf{C}(Y)$. The discrete valuation $v$ can be extended to a discrete valuation $v^{\prime}$ on $F$ which satisfies $v^{\prime}(f)=N v(f)$ for some integer $N$ and all $f \in \mathbf{C}(Y)$. One can then consider the action of $\pi_{1}(M)$ on the tree of $\mathrm{SL}_{2}(F)$ determined by the representation $P$. The fact that one of $v(\mathrm{l})$ or $v(\mathrm{~m})$ is non-zero implies that $P$ is not conjugate to a representation into $\mathrm{SL}_{2}\left(\mathcal{O}_{\mathrm{v}^{\prime}}\right)$ and hence that this action is non-trivial. Using transversality and Dehn surgery, exactly as in [CS1] or [CGLS], one obtains an incompressible surface in $M$.

Let us identify $\pi_{1}(\partial M)$ with a subgroup of $\pi_{1}(M)$ and with $H_{1}(\partial M)$. Then in our situation the unique slope $\{ \pm \gamma\}$ with the property that the trace of $P(\gamma)$ lies in the valuation ring $\mathcal{O}_{v^{\prime}}$ is that which corresponds to the extended rational number $-v(\mathrm{l}) / v(\mathrm{~m})$. As in [CGLS] this implies that the surface has non-empty boundary and that its boundary slope is $-v(\mathrm{l}) / v(\mathrm{~m})$.

3.2. Puiseaux parametrizations. Consider an irreducible plane curve $C$ defined by a homogeneous equation $F(x, y, z)=0$. Assume that $F$ is not divisible by $x$ or $y$ or $z$ and that $C$ passes through $(0: 0: 1)$. A Puiseaux parametrization of $C$ at $(0: 0: 1)$ is a solution of the equation $F(x, y, 1)=0$ in the power series ring $\mathbf{C}[[t]]$ of the form

$$
x(t)=t^{p} \quad \text { and } \quad y(t)=t^{q} \sum_{n=0}^{\infty} a_{n} t^{n}
$$

where $p$ and $q$ are positive integers and $a_{0} \neq 0$. Puiseaux proved that such solutions exist and that the power series $y(t)$ converges; thus a Puiseux parametrization does actually give a parametrization of the curve. (Puiseaux parametrizations exist at all points of $C$ but for our purposes it suffices to consider parametrizations at $(0: 0: 1)$.) Given a rational function $R(x, y)$ which represents a non-zero element of the function field of $C$, one defines the order of $R$ to be the integer $n$ such that $R(x(t), y(t))=t^{n} E(t)$ where $E(t)$ is a power series with non-zero constant term. It is shown in [Le] that the order depends only on the element of $\mathbf{C}(C)$ represented by $R$, and that the induced integer valued function on the non-zero elements of $\mathbf{C}(C)$ is a valuation. Note that if $v$ is the valuation determined by the Puiseaux parametrization 3.2.1 then $v(x)=p$ and $v(y)=q$, where we have abused notation by using $x$ and $y$ to denote the elements of $\mathbf{C}(C)$ represented by the corresponding polynomials. 
3.3. The Newton polygon. Given a polynomial

$$
F(x, y)=\sum_{m, n} b_{m n} x^{m} y^{n}
$$

consider the subset $\mathcal{N}_{F}$ of the integer lattice in $\mathbf{R}^{2}$ given by $\mathcal{N}_{F}=\left\{(m, n) \mid b_{m n} \neq 0\right\}$. The Newton polygon of $F$ is defined to be the convex hull of $\mathcal{N}_{F}$.

Let us assume that

$$
x(t)=t^{p} \quad \text { and } \quad y(t)=t^{q} \sum_{n=0}^{\infty} a_{n} t^{n}
$$

satisfy the equation $F(x, y)=0$. Let $f(m, n)$ be the linear functional on $\mathbf{R}^{2}$ given by $f(m, n)=m p+n q$. Setting the coefficient of the term of lowest degree $d$ in the power series $F(x(t), y(t))$ equal to 0 one obtains

$$
\sum_{f(m, n)=d} b_{m n} a_{0}^{n}=0
$$

Since $a_{0} \neq 0$, one obtains immediately that a necessary condition for $(x(t), y(t))$ to be a solution to $F(x, y)=0$ is that the minimum value of $f(m, n)$ on the set $\mathcal{N}(F)$ should be attained at two points. This is equivalent to the condition that the Newton polygon of $F$ have a side of slope $-p / q$ lying below $\mathcal{N}_{F}$. If this condition is satisfied then equation 3.3.1 exhibits $a_{0}$ as a root of a certain polynomial. It is proved in [Le] or [K] that if $a_{0}$ is taken to be any root of this polynomial then one can repeat this procedure to recursively determine the coefficients of $y(t)$, and that the power series produced in this way converges. This means that for each pair $(p, q)$ satisfying the necessary condition above there exists a Puiseaux parametrization of some irreducible component $C$ of the curve defined by $F$. In particular, for each side of the Newton polygon of $F$ which has slope $-p / q, p, q>0$, and lies below the set $\mathcal{N}_{F}$ there is a valuation $v$ on the function field of $C$ which satisfies $v(x)=p$ and $v(y)=q$. This statement is extended to apply to any side of the Newton polygon by the following proposition.

Proposition. Let $C$ be a plane curve with defining polynomial $F(x, y)$. Assume that $F$ is not divisible by $x$ or $y$. If the Newton polygon of $F$ has a side of slope $p / q$ then there is a valuation $v$ on the function field of some irreducible component of $C$ such that $p / q=-v(x) / v(y)$. Moreover we have $v(y)>0$ if and only if the side lies below $\mathcal{N}_{F}$.

ProOF. In view of the discussion above we need only show how to handle the case of a side of non-negative slope or a side which lies above $\mathcal{N}_{F}$.

If the Newton polygon of $F$ has side of slope 0 lying below $\mathcal{N}_{F}$ then there are at least two terms in $F$ which are powers of $x$. Thus there is a point on $C$ with coordinates $\left(x_{0}, 0\right)$ 
where $x_{0} \neq 0$. It is easy to see that if $v$ is a valuation on $\mathbf{C}(C)$ corresponding to a place centered at $\left(x_{0}, 0\right)$ then $v(x)=0$ while $v(y)>0$. A similar argument applies to the case where a side of the Newton polygon has slope $\infty$.

To handle the remaining cases one simply changes coordinates. Consider the birational self-equivalence of $\mathbf{C} P^{2}$ defined by the Cremona transformation $T(x: y: z)=\left(x y: z^{2}\right.$ : $y z)$. Note that if $y \neq 0$ then $T(x: y: 1)=(x: 1 / y: 1)$. In particular $T$ restricts to a birational isomorphism from the closure of $C$ to its image. The defining polynomial for $T(C)$ in the affine coordinates $x$ and $y$ is obtained from $F$ by substituting $1 / y$ for $y$ and multiplying by a power of $y$. The Newton polygon for this polynomial is obtained from that of $F$ by reflection through the horizontal axis and translation; the new polygon has a side of negative slope lying below it for each side of the Newton polygon of $F$ which has positive slope and lies above $\mathcal{N}_{F}$. If the Newton polygon for $F$ has a side of slope $p / q$, $p, q>0$, which lies above $\mathcal{N}_{F}$ then a Puiseaux parametrization for $T(C)$ gives rise to a valuation $v$ on the function field of some component of $T(C)$ with $v(x)=p$ and $v(y)=-q$. Since $T$ is a birational isomorphism from $C$ to $T(C)$ it induces isomorphisms between the function fields of corresponding components. This gives the proposition in this case. The other cases are handled the same way by using birational equivalences which map $(x: y: 1)$ to $(1 / x: y: 1)$ or $(1 / x: 1 / y: 1)$.

3.4. Theorem. The slopes of the sides of the Newton polygon of $A_{M}$ are boundary slopes of incompressible surfaces in $M$ which correspond to ideal points of $D_{M}$.

Proof. Combine Proposition 3.1 with Proposition 3.3.

\section{§4. Algebraic K-theory and $\tau_{v}(\mathrm{l}, \mathrm{m})$.}

The main result of this section implies that $\tau_{v}(l, m)$ is a root of unity for each valuation $v$ corresponding to an ideal point of $D_{M}$. As mentioned in the introduction, this implies that in the power series which appears in a Puiseaux parametrization of a local branch of $\overline{D_{M}}$ through a point at infinity, the leading coefficient is a root of unity. The proof given here uses results from algebraic K-theory about the group $K_{2}(F)$ where $F$ is a field with discrete valuation. In the next section we will give another proof of this fact using the tree of $\mathrm{SL}_{2}(F)$, which also relates the order of $\tau_{v}(\mathrm{l}, \mathrm{m})$ to topological information about incompressible surfaces in $M$.

The main result of the section can be summarized as follows. Let $Y$ denote the irreducible component of $D_{M}$ and $v$ the discrete valuation on $\mathbf{C}(Y)$ corresponding to the given ideal point. Let $F$ be the finite extension of $\mathbf{C}(Y)$ provided by Proposition 2.2, and 
let $F$ be endowed with a discrete valuation $v^{\prime}$ which extends the valuation $v$ on $\mathbf{C}(Y)$. The functions $l$ and $m$ determine an element $\{l, m\}$ of $K_{2}(F)$. The main result is that $\{l, m\}^{2}=0$. There is a homomorphism from $K_{2}(\mathbf{C}(Y))$ to $\mathbf{C}^{*}$, called the tame symbol, whose value on $\{l, m\}$ is a power of $\tau_{v}(l, m)$. Thus $\tau_{v}(l, m)$ is a root of unity.

Given units $f$ and $g$ in the coordinate ring of a smooth affine curve one can define a closed (real) differential 1-form on the curve by the formula $\eta(f, g)=\log |f| d \arg g-$ $\log |g| d \arg f$. This form arises in the construction of the regulator on $K_{2}$ of a curve. The main result of this section implies that the form $\eta(l, m)$ is exact on $Y$, a fact which has a geometric interpretation in terms of the volumes of hyperbolic dehn fillings of $M$. For an arbitrary curve in $\mathbf{C}^{*} \times \mathbf{C}^{*}$, the exactness of $\eta(\mathrm{l}, \mathrm{m})$ gives a computable obstruction to realizing the curve as $D_{M}$ for a 3-manifold $M$.

4.1. The symbol. Suppose that $\Lambda$ is a commutative ring. Let $\operatorname{SL}(\Lambda)$ denote the direct limit of the groups $\mathrm{SL}(\mathrm{n}, \Lambda)$ and $\mathrm{E}(\Lambda)<\mathrm{SL}(\Lambda)$ the direct limit of the groups $\mathrm{E}(\mathrm{n}, \Lambda)$ of $n \times n$ elementary matrices. Recall that the abelian group $K_{2}(\Lambda)$ is canonically isomorphic to the kernel of the universal central extension of $\mathrm{E}(\Lambda)$. The extension is the Steinberg group $\operatorname{St}(\Lambda)$. In other words we have a short exact sequence

$$
0 \longrightarrow K_{2}(\Lambda) \longrightarrow \mathrm{St}(\Lambda) \longrightarrow \mathrm{E}(\Lambda) \longrightarrow 0
$$

which is a universal central extension. Recall also that it follows from the five-term exact sequence of a group extension that the kernel of a universal central extension is naturally isomorphic to the second integral homology group of the quotient. So we have a natural isomorphism $\eta: K_{2}(\Lambda) \rightarrow H_{2}(\mathrm{E}(\Lambda))$.

Following Milnor [M], given any two commuting elements $U$ and $V$ of $\mathrm{E}(\Lambda)$ we define an element $U \star V$ of $K_{2}(\Lambda)$ by lifting $U$ and $V$ to elements $u$ and $v$ of $\operatorname{St}(\Lambda)$ and setting $U \star V=u v u^{-1} v^{-1}$. This commutator lies in the kernel of the central extension, since $U$ and $V$ commute, and is independent of the choice of lifts.

Given any two units $f$ and $g$ in $\Lambda$ the symbol $\{f, g\}$ is an element of $K_{2}(\Lambda)$ which is defined as follows. Consider the matrices

$$
D_{f}=\left[\begin{array}{ccc}
f & 0 & 0 \\
0 & f^{-1} & 0 \\
0 & 0 & 1
\end{array}\right] \text { and } D_{g}^{\prime}=\left[\begin{array}{ccc}
g & 0 & 0 \\
0 & 1 & 0 \\
0 & 0 & g^{-1}
\end{array}\right]
$$

which are elements of $\mathrm{E}(3, \Lambda)$. Then $\{f, g\}=D_{f} \star D_{g}^{\prime}$.

By [M, Lemma 8.3], using multiplicative notation for the abelian group $K_{2}$ and writing $\operatorname{diag}\left(f_{1}, \ldots, f_{n}\right)$ for the diagonal matrix with diagonal entries $f_{1}, \ldots, f_{n}$, we have

$$
\operatorname{diag}\left(f_{1}, \ldots, f_{n}\right) \star \operatorname{diag}\left(g_{1}, \ldots, g_{n}\right)=\left\{f_{1}, g_{1}\right\} \ldots\left\{f_{n}, g_{n}\right\} .
$$

Thus we have $\operatorname{diag}\left(f, f^{-1}\right) \star \operatorname{diag}\left(g, g^{-1}\right)=\{f, g\}\left\{f^{-1}, g^{-1}\right\}=\{f, g\}^{2}$. (The last equality follows from the bimultiplicativity of the symbol [M, Lemma 8.2].) 
Lemma. Suppose that $f$ and $g$ are units in $\Lambda$. Let $A$ be a free abelian group with basis $\{U, V\}$. Let $\phi: A \rightarrow \mathrm{E}(\Lambda)$ be the homomorphism defined by

$$
\phi(U)=\operatorname{diag}\left(f, f^{-1}\right) \quad \text { and } \quad \phi(V)=\operatorname{diag}\left(g, g^{-1}\right) .
$$

Then there is a generator $\xi$ of $H_{2}(A)$ such that $\eta_{*} \circ \phi_{*}(\xi)=\{f, g\}^{2}$.

Proof. By the remarks above it suffices to show that

$$
\eta_{*} \circ \phi_{*}(\xi)=\phi(U) \star \phi(V) .
$$

Let $F$ be a free group on the letters $U$ and $V$. The homomorphism $\phi$ determines a commutative diagram

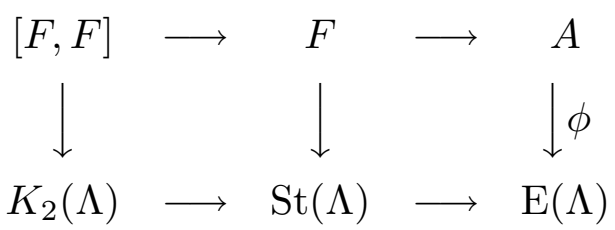

for which the homomorphism from $[F, F]$ to $K_{2}(\Lambda)$ sends the commutator $[U, V]$ to $\phi(U) \star \phi(V)$. Consider the commutative diagram below in which the vertical maps between terms of the five-term exact sequences are induced by $\phi$.

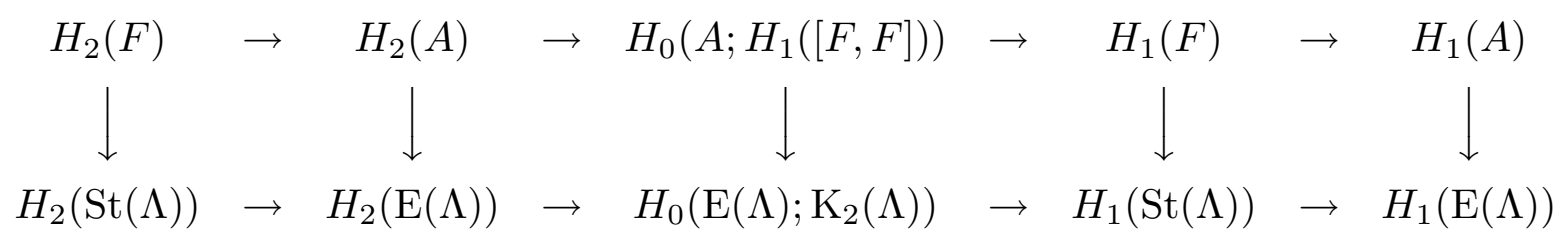

Note that the second map is an isomorphism in each of the five-term sequences. If we identify $H_{0}\left(A ; H_{1}([F, F])\right.$ with the second quotient of the lower central series of $F$, which is a cyclic group generated by the coset of $[U, V]$, then the isomorphism from $H_{2}(A)$ to $H_{0}\left(A ; H_{1}([F, F])\right.$ must take a generator of $H_{2}(A)$ to the coset of $[U, V]$. Since $[U, V]$ maps to $\phi(U) \star \phi(V)$ in $K_{2}(\Lambda)=H_{0}\left(\mathrm{E}(\Lambda) ; \mathrm{H}_{1}\left(\mathrm{~K}_{2}(\Lambda)\right)\right)$, the lemma follows from the commutativity of the diagram.

Proposition. Suppose that $M$ is a 3-manifold with boundary a torus and that $\mathrm{M}$ and $\mathrm{L}$ form a basis of the peripheral subgroup of $M$. Suppose that $\rho$ is a representation of $\pi_{1}(M)$ into $\mathrm{SL}_{2}(\Lambda)$ for which $\rho(\mathrm{L})=\operatorname{diag}\left(l, \mathrm{l}^{-1}\right)$ and $\rho(\mathrm{M})=\operatorname{diag}\left(\mathrm{m}, \mathrm{m}^{-1}\right)$. Then the symbol $\{l, \mathfrak{m}\} \in K_{2}(\Lambda)$ has order dividing 2 .

Proof. The homomorphism induced by $\rho$ from $H_{2}\left(\pi_{1}(\partial M)\right)$ to $H_{2}(\mathrm{E}(\mathrm{F}))=\mathrm{K}_{2}(\mathrm{~F})$ factors through the homomorphism induced on $H_{2}$ by the inclusion of $\pi_{1}(\partial M)$ into $\pi_{1}(M)$ and is thus trivial. 
4.2. The tame symbol. Here we consider representations of $\pi_{1}(M)$ into $\mathrm{SL}_{2}(F)$, where $F$ is a field with discrete valuation $v$ and residue field $k$.

Recall [M, Corollary 9.13] that $K_{2}(F)$ is generated by the symbols $\{f, g\}$ for $f, g \in F^{*}$. Recall also that there is a homomorphism $d_{v}: K_{2}(F) \rightarrow k^{*}$ which is called the tame symbol and is defined by

$$
d_{v}(\{f, g\})=f^{v(g)} / g^{v(f)} .
$$

(The element $f^{v(g)} / g^{v(f)}$ is a unit in $\mathcal{O}$ and hence has a well-defined value in $k^{*}$. By definition this value is $\tau_{v}(f, g)^{s}$, where $\left.s=(v(f), v(g))\right)$.

Suppose now that $F$ is the function field of a smooth projective curve $C$ and that $v$ is the valuation associated to a point $p$ of $C$. Let $\rho$ be as above, so that for a general peripheral element $\mathrm{L}^{m} \mathrm{M}^{n}$ we have

$$
\rho\left(\mathrm{L}^{m} \mathrm{M}^{n}\right)=\left[\begin{array}{cc}
\mathrm{l}^{m} \mathrm{~m}^{n} & 0 \\
0 & \mathrm{l}^{-m} \mathrm{~m}^{-n}
\end{array}\right] .
$$

Unless $l$ and $m$ are both finite-valued at $p$ there will be a primitive element $\gamma$ of the peripheral subgroup of $\pi_{1}(M)$, unique up to inverse, for which the diagonal entries of $\rho(\gamma)$ are finite-valued at $p$. We must have

$$
\gamma^{ \pm s}=\mathrm{L}^{v(\mathrm{~m})} / \mathrm{M}^{v(\mathrm{l})}
$$

where $s$ is the greatest common divisor of $v(\mathrm{l})$ and $v(\mathrm{~m})$. Since the tame symbol is a homomorphism, Proposition 4.1 implies that the eigenvalues of $\gamma(p)$ are roots of unity whose order divides $2 s$.

4.3. Let $x$ be an ideal point of $D_{M}$ and $Y$ an irreducible component of $D_{M}$ such that $x$ is contained in $\tilde{Y}$. Let $F$ be the finite extension of $\mathbf{C}(Y)$ and $P$ the representation provided by Proposition 2.2. Let $v$ be the discrete valuation on $\mathbf{C}(Y)$ associated to $x$ and $v^{\prime}$ a discrete valuation on $F$ satisfying $\left.v^{\prime}\right|_{\mathbf{C}(Y)^{*}}=N v$.

Proposition. Let $s$ be the greatest common divisor of $v(\mathrm{l})$ and $v(\mathrm{~m})$. If the incompressible surfaces in $M$ associated to the ideal point $x$ of $D_{M}$ have boundary slope $p / q$ then $\tau_{v}(\mathrm{l}, \mathrm{m})$ is a root of unity of order dividing $2 \mathrm{Ns}$.

Proof. Let $d_{v^{\prime}}: K_{2}(F) \rightarrow C^{*}$ and $d_{v}: K_{2}(\mathbf{C}(Y)) \rightarrow C^{*}$ be the tame symbols. Note that $d_{v^{\prime}}(\{l, \mathfrak{m}\})=d_{v}(\{l, \mathfrak{m}\})^{N}$. Thus by 4.2 we have

(1) $d_{v^{\prime}}(\{l, \mathfrak{m}\})$ is a root of unity of order dividing 2 ;

(2) $d_{v}(\{l, \mathfrak{m}\})$ is a root of unity of order dividing $2 N$;

(3) $\tau_{v}(l, m)$ is a root of unity of order dividing $2 N s$. 
4.4. Regulator homomorphisms and the 1-form $\eta(\mathrm{l}, \mathrm{m})$. In this section and the next we describe some connections with the hyperbolic geometry of $M$. These sections are independent of the rest of the paper.

Suppose that $Y$ is a smooth complex projective curve and that $f$ and $g$ are functions on $Y$. Let $S$ be a finite subset of $Y$ containing the zeroes and poles of both $f$ and $g$. We then define a (real) differential 1-form on $Y-S$ by the formula

$$
\eta(f, g)=\log |f| d \arg g-\log |g| d \arg f
$$

Note that

$$
d \eta=\Im\left(\frac{d f}{f} \wedge \frac{d g}{g}\right)
$$

Thus $d \eta$ is the imaginary part of a complex 2-form on $Y$, so $\eta(f, g)$ is a closed 1-form. We denote by $c(f, g)$ the class in $H^{1}(Y-S ; \mathbf{R})$ whose value on the homology class represented by a loop $\gamma$ in $Y-S$ is given by $\frac{1}{2 \pi} \int_{\gamma} \eta(f, g)$. We will regard $c(f, g)$ as an element of the direct limit of the groups $H^{1}(Y-S ; \mathbf{R})$ as $S$ ranges over the finite subsets of $Y$.

Proposition. There is a unique homomorphism $r_{0}: K_{2}(\mathbf{C}(Y)) \rightarrow \lim H^{1}(Y-S ; \mathbf{R})$ which sends the symbol $\{f, g\}$ to $c(f, g)$.

Proof. Using Matsumoto's Theorem, it suffices (see [M, Corollary 1.3]) to show that $c(f, g)$ satisfies

(i) $c\left(f_{1} f_{2}, g\right)=c\left(f_{1}, g\right)+c\left(f_{2}, g\right)$

(ii) $c(f, g)=-c(g, f)$

(iii) $c(f, 1-f)=0$

The first two properties are immediate, so we concentrate on property (iii). Let $S$ be a finite subset of $Y$ such that neither $f$ nor $1-f$ has a zero or a pole in $Y-S$. We may then regard $f$ as a function from $Y-S$ to $\mathbf{P}^{1}-\{0,1, \infty\}$. By naturality it suffices to check (iii) in the case where $Y=\mathbf{P}^{1} \subset \mathbf{C}, S=\{0,1, \infty\}$, and $f=z$ is the standard coordinate. If $\gamma$ is a circle of radius $\epsilon$ about 0 then

$$
\int_{\gamma} \eta(z, 1-z)=\int_{\gamma} \log |z| d \arg (1-z)-\int_{\gamma} \log |1-z| d \arg (z)
$$

Now,

$$
\int_{\gamma} \log |z| d \arg (1-z)=\epsilon \int_{\gamma} d \arg (1-z)=0
$$

and

$$
\left|\int_{\gamma} \log \right| 1-z|d \arg (z)|<2 \pi|\log (1-\epsilon)|
$$


Taking the limit as $\epsilon \rightarrow 0$ shows that $c(z, 1-z)$ takes the value 0 on the homology class represented by $\gamma$. A symmetric calculation about 1 shows that $c(z, 1-z)$ vanishes on a basis of $H_{1}\left(\mathbf{P}^{1}-\{0,1, \infty\} ; \mathbf{R}\right)$, proving the proposition.

Combining the proposition above with Proposition 4.1 gives:

Corollary. The 1-form $\eta(\mathrm{l}, \mathrm{m})$ is exact on the smooth projective model of each irreducible component of the curve $D_{M}$.

Given a curve in $\mathbf{C}^{*} \times \mathbf{C}^{*}$, the exactness of $\eta(l, m)$ is a computable obstruction to realizing the curve as $D_{M}$ for a 3-manifold $M$; integration of $\eta(l, m)$ around a cycle in the complement of the singular set of the curve must yield 0 if the curve is to be realizable as $D_{M}$.

The homomorphism $r$ is very closely related to the regulator homorphism on $K_{2}$ of a curve. Specifically, it follows from $[\mathrm{R}, 4.2,4.4$, and 6.2] that if $Y$ is defined over a number field $k \subset \mathbf{C}$ then there is a commutative diagram

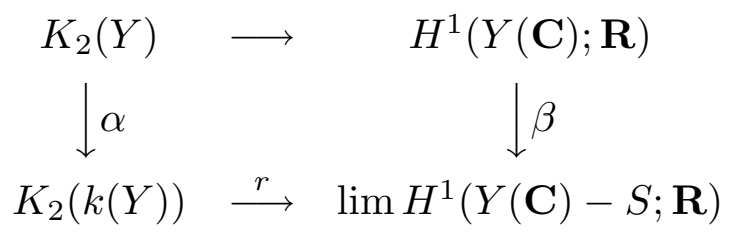

where $\alpha$ is the localization homomorphism, $\beta$ is the injection induced by inclusion and the map at the top is, up to twisting by $2 \pi i$, the regulator as defined by Beilinson.

4.5. Volumes. The exactness of $\eta(\mathrm{l}, \mathrm{m})$ has geometric significance. We will explain, following Hodgson $[\mathrm{H}]$, how to define a natural "volume" function $V: R(M) \rightarrow \mathbf{R}$ which has the property that if $\rho$ is a discrete faithful representation of $\pi_{1}(M)$ then $V(\rho)$ is the volume of the hyperbolic manifold $\rho\left(\pi_{1}(M)\right) \backslash \mathbf{H}^{3}$. The differential $d V$ is essentially a pull-back of the 1-form $\eta(\mathrm{l}, \mathrm{m})$ to $R(M)$, which implies the exactness of $\eta$.

For the definition of the function $V$ we begin with a triangulation of the 1-point compactification of $M-\partial M$. Deleting the point at infinity produces a triangulation of $M$ with some ideal vertices. Lift this triangulation to the universal cover of $M$ and add points at infinity to complete the simplices with ideal vertices. The result is a simplicial complex which we shall denote $\tilde{M}$. There is a simplicial action of $\pi_{1}(M)$ on this complex for which each interior vertex has a trivial stabilizer while each vertex at infinity is stabilized by a conjugate of the peripheral subgroup of $\pi_{1}(M)$.

Let $\rho: \pi_{1}(M) \rightarrow \mathrm{SL}_{2}(\mathbf{C})$ be a representation. Let $\overline{\mathbf{H}}^{3}$ denote hyperbolic space compactified by adjoining the sphere at infinity. A map of a 3-simplex into $\overline{\mathbf{H}}^{3}$ will be called totally geodesic if its image is a hyperbolic 3 -simplex with totally geodesic faces and if it 
sends faces to faces. (The hyperbolic simplex is allowed to have vertices at infinity and may be degenerate.) By a pseudo-hyperbolic structure with holonomy $\rho$ we will mean a map from $\tilde{M}$ to $\overline{\mathbf{H}}^{3}$ which is $\pi_{1}(M)$-equivariant with respect to the action determined by $\rho$, sends vertices at infinity of $M$ to points at infinity in $\overline{\mathbf{H}}^{3}$, and restricts to a totally geodesic map on each 3 -simplex. Such a map is not necessarily an immersion since it is not required to preserve orientation of the 3 -simplices. If $\Psi$ is a pseudo-hyperbolic structure then the volume of $\Psi$ is defined to be $\sum_{\sigma \in \Sigma} \epsilon(\sigma)$ vol $\Psi(\sigma)$ where $\Sigma$ is a complete set of representatives for the $\pi_{1}(M)$-orbits of 3 -simplices in $\tilde{M}$, and $\epsilon(\sigma)= \pm 1$ according to whether $\Psi$ preserves the orientation of $\sigma$.

Given a representation $\rho$, it is not difficult to construct a pseudo-hyperbolic structure with holonomy $\rho$. Choose representatives $v_{1}, v_{2}, \ldots, v_{n}$ of the $\pi_{1}(M)$-orbits of the interior vertices of $\tilde{M}$. The action of $\pi_{1}(M)$ is transitive on the vertices at infinity of $\tilde{M}$; let $v_{\infty}$ be a representative of this orbit. The stabilizer of $v_{\infty}$ is a conjugate of the peripheral subgroup and has a common fixed point on the sphere at infinity of hyperbolic space. Map $v_{\infty}$ to such a fixed point and map $v_{1}, \ldots, v_{n}$ to arbitrary interior points of $\mathbf{H}^{3}$. There is a unique extension to a $\pi_{1}(M)$-equivariant map of the 0 -skeleton of $\tilde{M}$. This can be extended over the 1-skeleton, sending edges to geodesic segments, and over the 2-skeleton, sending faces to totally geodesic triangles. Finally, the map can then be extended over the 3 -simplices to give a pseudo-hyperbolic structure. More generally, given a smooth 1-parameter family $\rho_{t}$ of representations, it is clearly possible to construct a smooth 1-parameter family $\Psi_{t}$ of pseudo-hyperbolic structures so that the holonomy of $\Psi_{t}$ is $\rho_{t}$.

Now, using Schläfli's formula for the derivative of the volume of a smooth 1-parameter family of hyperbolic polyhedra, Hodgson computes in $[\mathrm{H}]$ the derivative of the volume of a smooth family of pseudo-hyperbolic structures on $M$. Let $\Psi_{t}$ be such a family, let $\rho_{t}$ be the holonomy of $\Psi_{t}$, and let $l_{t}$ and $m_{t}$ be the eigenvalues of $\rho_{t}(\mathrm{~L})$ and $\rho_{t}(M)$ associated to a common eigenvector. We can take $\rho_{t}, l_{t}$ and $m_{t}$ to depend smoothly on $t$. In this situation, Hodgson's calculation shows that

$$
\frac{d V}{d t}=-\frac{1}{2}\left(\log |l| \frac{d \arg \mathrm{m}}{d t}-\log |\mathrm{m}| \frac{d \arg l}{d t}\right)
$$

This implies that the function $V$ determines a well-defined function on $D_{M}$ which is smooth away from the singularities. Lifting to the smooth projective model of an irreducible component of $D_{M}$ we obtain a smooth function whose differential is $\eta(\mathrm{l}, \mathrm{m})$.

A slight extension is required to apply Hodgson's argument in our situation since he only considers the case where the pseudo-hyperbolic structures are immersions and where the triangulation has only ideal vertices. It is remarked in $[\mathrm{H}]$ that the arguments apply without change to the case of pseudo-hyperbolic structures which are not immersions. The 
Schläfli formula states that for a smooth family of polyhedra in $\mathbf{H}^{3}$ the differential of the volume is given by

$$
d V=-\frac{1}{2} \sum l_{i} d \theta_{i}
$$

where the sum is over the edges of the polyhedron, and $l_{i}$ and $\theta_{i}$ denote respectively the length and dihedral angle of the $i^{\text {th }}$ edge. In the case of a polyhedron with ideal vertices one first chooses an arbitrary small horosphere at each ideal vertex along which to truncate the polyhedron. The value of $l_{i}$ is interpreted to be the length of the truncated edge; the choice of truncating horosphere does not affect the differential since the sum of the dihedral angles at the edges incident to an ideal vertex is constant. In computing the differential of the volume of a pseudo-hyperbolic structure one obtains a sum of contributions from the edges of the triangulation. Edges with ideal vertices make non-trivial contributions, which reflect the fact that in general it is not possible to choose invariant horospheres at the ideal points when truncating the simplices of the triangulation. This part of Hodgson's argument goes through in our setting. One must only add the observation that an edge with no ideal endpoints contributes 0 because the sum of the dihedral angles of the simplices containing the edge is constant.

\section{$\S 5$. The tree of $\mathrm{SL}_{2}(F)$ and $\tau_{v}(l, m)$.}

We again consider an ideal point $x$ of $D_{M}$. Let $Y$ be the component of $D_{M}$ and $v$ the discrete valuation on $\mathbf{C}(Y)$ associated to $x$. We have seen that there is an incompressible surface $S$ in $M$ with boundary slope $-v(\mathrm{l}) / v(\mathrm{~m})$ which is also associated to $x$. Assume that $S$ has been chosen among all such surfaces to have the fewest possible number of boundary components. The main result of this section shows that the root of unity $\tau_{v}(\mathrm{l}, \mathrm{m})$ carries topological information about this surface. We show that the order of $\tau_{v}(l, m)$ divides the number of boundary components of any component of $S$.

The main theorem of this section, Theorem 5.7, applies to a general representation $P$ of $\pi_{1}(M)$ in $\mathrm{SL}_{2}(F)$, where $F$ is a field with discrete valuation. The application to $D_{M}$, Corollary 5.7, is obtained by specializing to the case where $F$ is the finite extension of the function field of an irreducible component of $D_{M}$ provided by Proposition 2.2.

At the end of this section we state the conditions which are imposed upon the coefficients of $A_{M}$ by the conclusion of Theorem 5.7.

5.1. Eigenvalues. Let $F$ be a field with discrete valuation $v: F^{*} \rightarrow \mathbf{Z}$. We denote by $\mathcal{O}$ the valuation ring of $F$, by $\pi$ a generator of the maximal ideal in $\mathcal{O}$ and by $k$ the residue field $\mathcal{O} /(\pi)$.

Recall from [S] that the affine building $T_{F}$ of $\mathrm{SL}_{2}(F)$ is a tree. The vertices of $T_{F}$ are homothety classes of $\mathcal{O}$-lattices in the 2 -dimensional vector space $F^{2}$. There is an edge of $T_{F}$ joining the class $[L]$ to the class $\left[L^{\prime}\right]$ whenever $\pi L \varsubsetneqq L^{\prime} \varsubsetneqq L$. By "edge" we shall 
always mean "directed edge"; there are two edges joining each pair of adjacent vertices, one in each direction. If $e$ is an edge then $\bar{e}$ will denote the opposite of $e$, the edge with the same endpoints and opposite orientation. We denote by $i(e)$ and $t(e)$ respectively the initial and terminal vertices of an edge $e$. The set of edges of $T_{F}$ with initial vertex $[L]$ are in one-to-one correspondence with the lines in the 2 -dimensional $k$-vector space $L / \pi L$. If $L^{\prime}$ is an $\mathcal{O}$-lattice with $\pi L \varsubsetneqq L^{\prime} \varsubsetneqq L$ then the edge from $[L]$ to $\left[L^{\prime}\right]$ corresponds to the 1-dimensional subspace $L^{\prime} / \pi L$ of $L / \pi L$.

The action of $\mathrm{SL}_{2}(F)$ on the set of $\mathcal{O}$-lattices in $F^{2}$ induces an action on $T_{F}$. This is an action without inversions, i.e. no element of $\mathrm{SL}_{2}(F)$ sends an edge to its opposite. The stabiliizer in $\mathrm{SL}_{2}(F)$ of a vertex $[L]$ coincides with the stabilizer of the $\mathcal{O}$-lattice $L$, and is therefore conjugate in $\mathrm{GL}_{2}(F)$ to $\mathrm{SL}_{2}(\mathcal{O})$. It follows (cf. [CS1, Theorem 2.2.1] that $A$ fixes some vertex of $T_{F}$ if and only if $\operatorname{tr} A \in \mathcal{O}$. Let $e$ be an edge of $T$ which is fixed by an element $A \in \mathrm{SL}_{2}(F)$. Then $i(e)$ and $t(e)$ are represented by lattices $L$ and $L^{\prime}$ such that $\pi L \varsubsetneqq L^{\prime} \varsubsetneqq L$; the linear transformation of the 1-dimensional $k$-vector space $L^{\prime} / \pi L$ which is induced by $A$ must be multiplication by a scalar $\lambda$. We shall call $\lambda$ the eigenvalue of $A$ associated to the fixed edge $e$. The eigenvalue $\lambda$ does not depend on the choice of $L$ and $L^{\prime}$ because the pair $\left\{L, L^{\prime}\right\}$ is uniquely determined up to homothety.

Note that we may interpret $\lambda$ as the eigenvalue corresponding to the eigenspace $L^{\prime} / \pi L$ of the linear transformation induced by $A$ on the 2-dimensional vector space $L / \pi L$.

Note also that if $A \in \mathrm{SL}_{2}(F)$ fixes an edge then it necessarily fixes two vertices, so its trace lies in $\mathcal{O}$. If $\lambda$ is the eigenvalue of $A$ associated to the edge then $\lambda+\lambda^{-1}$ is the image of $\operatorname{tr} A$ under the quotient map $\mathcal{O} \rightarrow \mathcal{O} / \pi=k$.

Finally, note that the eigenvalue of $A$ associated to a fixed edge $e$ depends only on the conjugacy class of $A$ in the stabilizer of $e$.

5.2. Proposition. Suppose that $A \in \mathrm{SL}_{2}(F)$ fixes an edge $e$ of $T_{F}$. If the eigenvalue of $A$ associated to $e$ is $\lambda$ then the eigenvalue of $A$ associated to $\bar{e}$ is $\lambda^{-1}$.

Proof. Let the initial and terminal vertices of $e$ be $[L]$ and $\left[L^{\prime}\right]$ respectively, where $\pi L \varsubsetneqq L^{\prime} \varsubsetneqq L$. Then $A$ induces multiplication by $\lambda$ on $L^{\prime} / \pi L$. On the other hand we have $\pi L^{\prime} \varsubsetneqq \pi L \varsubsetneqq L^{\prime}$; hence if $\bar{\lambda}$ denotes the eigenvalue of $A$ associated to $\bar{e}$, then $A$ induces multiplication by $\bar{\lambda}$ on $\pi L / \pi L^{\prime} \cong L / L^{\prime}$. From the exact sequence

$$
0 \rightarrow L^{\prime} / \pi L \rightarrow L / \pi L \rightarrow L / L^{\prime} \rightarrow 0
$$

it follows that $\lambda \bar{\lambda}$ is the determinant of the linear transformation induced by $A$ on $L / \pi L$. Since $A \in \mathrm{SL}_{2}(F)$ this means that $\bar{\lambda}=\lambda^{-1}$. 
5.3. By an edge path in $T_{F}$ we mean a sequence $e_{0}, \ldots, e_{n}$ of edges of $T_{F}$ with $i\left(e_{j}\right)=$ $t\left(e_{j-1}\right)$ for $j=1, \ldots, n$. The edge path $e_{0}, \ldots, e_{n}$ is reduced if $e_{j} \neq \bar{e}_{j-1}$ for $j=1, \ldots, n$. We define a partial ordering of the edges of $T_{F}$ by setting $e \leq e^{\prime}$ if there is a reduced edge path $e=e_{0}, \ldots, e_{n}=e^{\prime}$. For any pair $\left\{e, e^{\prime}\right\}$ of distinct edges exactly one of the following relations holds: $e<e^{\prime}, \bar{e}<e^{\prime}, e^{\prime}<e$, or $e^{\prime}<\bar{e}$.

Proposition. Let $A$ be an element of $\mathrm{SL}_{2}(F)$ which fixes two edges $e$ and $e^{\prime}$ of $T_{F}$. The eigenvalues of $A$ associated to $e$ and $e^{\prime}$ are equal if $e<e^{\prime}$ or $e^{\prime}<e$. They are reciprocals if $\bar{e}<e^{\prime}$ or $e^{\prime}<\bar{e}$.

Proof. We will assume that $e<e^{\prime}$; the other cases follow from this one by Proposition 5.2.

Let $e_{0}, \ldots, e_{n}$ be the unique reduced edge path with $e=e_{0}$ and $e_{n}=e^{\prime}$. Since $A$ fixes $e$ and $e^{\prime}$ it must fix all of the $e_{i}$. Let $\lambda$ be the eigenvalue of $A$ associated to $e$. By Proposition 5.2, $\lambda^{-1}$ is the eigenvalue associated to $\bar{e}$.

Now $\bar{e}_{0}$ and $e_{1}$ are distinct edges with the same initial vertex $[L]$ and terminal vertices $\left[L_{0}\right]$ and $\left[L_{1}\right]$, where $\pi L \varsubsetneqq L_{0} \varsubsetneqq L$ and $\pi L \varsubsetneqq L_{1} \varsubsetneqq L$. The 1-dimensional subspaces $L_{0} / \pi L$ and $L_{1} / \pi L$ of $L / \pi L$ are invariant under the linear transformation of $L / \pi L$ induced by $A$; furthermore these subspaces are distinct and therefore span $L / \pi L$. Since $A$ has determinant 1 and induces multiplication by $\lambda^{-1}$ on $L_{0} / \pi L$, it must induce multiplication by $\lambda$ on $L_{1} / \pi L$. This means that the eigenvalue associated to $e_{1}$ is $\lambda$. It now follows by induction that the eigenvalue of $A$ associated to $e^{\prime}=e_{n}$ is also $\lambda$.

5.4. The statement of Proposition 5.3 implies that if $\operatorname{tr} A \neq \pm 2$ then comparability is an equivalence relation on the set of fixed edges of $A$. This may appear paradoxical because comparability is never an equivalence relation on the set of edges of a tree which contains a vertex of valence at least three: consider the three edges shown in Figure 5.4.1 where $e_{0}$ is comparable to $e_{1}$ and to $e_{2}$ but $e_{1}$ and $e_{2}$ are not comparable.

However, it is not hard to show directly that if $A$ fixes at least one edge of $T_{F}$ and $\operatorname{tr} A \neq \pm 2$ then the fixed point set of $A$ is a line.

5.5. Reduced surfaces. We now return to our compact, orientable, 3-manifold $M$ with $\partial M$ a torus. We fix a universal covering $p: \tilde{M} \rightarrow M$ and write $\pi_{1}(M)$ for the group of deck transformations of $M$. We suppose that we are given a representation $P: \pi_{1}(M) \rightarrow \mathrm{SL}_{2}(F)$. Then $P$ determines an action of $\pi_{1}(M)$ on $T_{F}$, and the fundamental group of any connected submanifold of $M$ has an induced action defined up to conjugacy. We assume that the induced action of $\pi_{1}(\partial M)$ on $T_{F}$ does not fix any vertex.

In this situation the theory developed in [CS1], as re-formulated in [CGLS], allows one to associate properly embedded essential surfaces to the action of $\pi_{1}(M)$ on $T_{F}$. We 


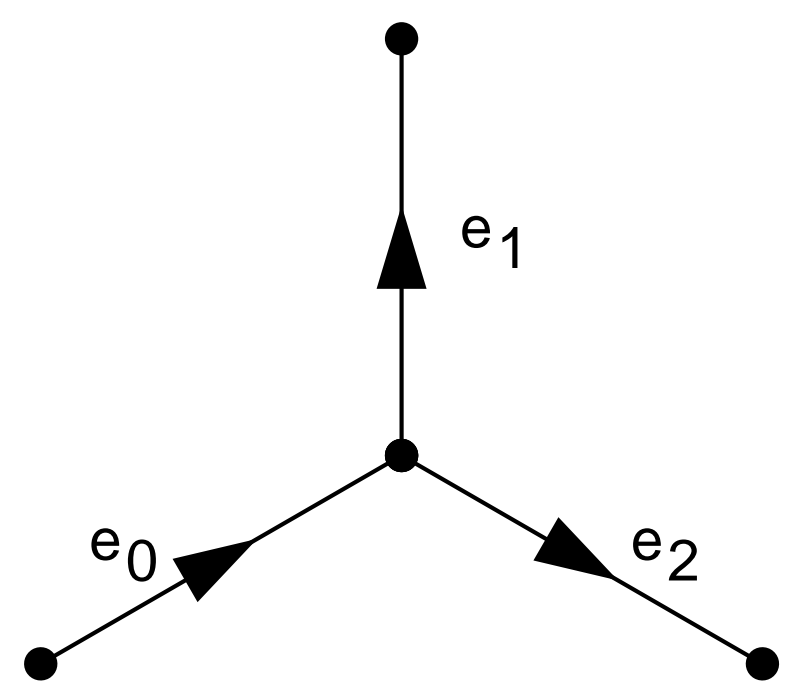

Figure 5.4.1

review the relevant definition. Let $E$ denote the set of midpoints of edges of $T_{F}$. For $S$ to be associated to the action of $\pi_{1}(M)$ on $T_{F}$ means that there is a $\pi_{1}(M)$-equivariant map $\tilde{\phi}: \tilde{M} \rightarrow T$ such that $\tilde{\phi}^{-1}(E)=p^{-1}(S)$.

A surface $S$ associated to the action of $\pi_{1}(M)$ on $T_{F}$ is not necessarily connected. The assumption that $\pi_{1}(M)$ fixes no vertex of $T_{F}$ implies that $\partial S \neq \emptyset$.

A surface associated to an action of $\pi_{1}(M)$ on $T_{F}$ will be said to be reduced if it is essential and has the minimal number of boundary components among all essential surfaces associated to the action.

5.6. Fix a reduced surface $S$ associated to the action of $\pi_{1}(M)$ on $T_{F}$. Also fix orientations of $M$ and $S$. Then the components of $\partial \tilde{M}$ and of $p^{-1}(S)$ have induced orientations, and the components of $S$ and $\partial S$ have induced transverse orientations in $M$ and $\partial M$ respectively.

Under our assumptions either $\partial M$ is incompressible or some component of $S$ is a boundary compressing disk. Otherwise the image of $\pi_{1}(\partial M)$ in $\pi_{1}(M)$ would be a cyclic subgroup containing an element of the non-trivial conjugacy class represented by a component of $\partial S$. This cyclic group would contain a non-trivial element which fixed a vertex of $T_{F}$. This implies that the entire cyclic group fixes a vertex and hence that there is a vertex fixed by $\pi_{1}(\partial M)$ under the induced action, contrary to our assumption.

If $\partial M$ is incompressible then the components of $\partial \tilde{M}$ are planes and the components of $p^{-1}(\partial S)$ contained in a single component of $\partial \tilde{M}$ form a family of parallel lines. If $\partial M$ is compressible then the components of $\partial \tilde{M}$ are open annuli and the components of $p^{-1}(\partial S)$ contained in a single component of $\partial \tilde{M}$ form a family of parallel essential circles. We will say that two components of $p^{-1}(\partial S)$ have compatible orientations if they project to homologous oriented simple closed curves in the torus $\partial M$. 
For each component $\tilde{C}$ of $p^{-1}(\partial S)$ there is a unique (directed) edge $e(\tilde{C})$ of $T_{F}$ so that $\phi(\tilde{C})$ is the midpoint of $e(\tilde{C})$ and the direction of $e(\tilde{C})$ pulls back to the transverse orientation of $\tilde{C}$.

Proposition. Under the above assumptions, suppose that $\tilde{C}$ and $\tilde{C}^{\prime}$ are components of $p^{-1}(\partial S)$ which lie in the same component of $\partial \tilde{M}$. Then the orientations of $\tilde{C}$ and $\tilde{C}^{\prime}$ are compatible if and only if the edges $e(\tilde{C})$ and $e\left(\tilde{C}^{\prime}\right)$ are comparable.

Proof. Let $U$ be the component of $\partial \tilde{M}$ containing $C$ and $C^{\prime}$.

First consider the case where $C$ and $C^{\prime}$ are adjacent on $U$. We claim that in this case $\tilde{\phi}(C) \neq \tilde{\phi}\left(C^{\prime}\right)$, i.e. that $e(C) \neq e\left(C^{\prime}\right)$ and $e(C) \neq \overline{e\left(C^{\prime}\right)}$.

The proof of this claim is a simple application of the techniques used in Section 1.3 of [CGLS]. Suppose that $\tilde{\phi}(C)=\tilde{\phi}\left(C^{\prime}\right)$. Let $\tilde{A}$ denote the submanifold of $U$ which is bounded by $C$ and $C^{\prime}$. Note that $p$ restricts to a covering projection from $\tilde{A}$ to an annulus $A \subset \partial M$ which is bounded by two adjacent components of $\partial S$. Since $p^{-1}(S)=\tilde{\phi}^{-1}(E)$, the components $C$ and $C^{\prime}$ of $\tilde{\phi}^{-1}(E)$ are mapped by $\tilde{\phi}$ to the same point of $E$ while the interior of $\tilde{A}$ maps into a (contractible) component of $T_{F}-E$. Thus the map $\tilde{\phi}$ can be modified in a small neighborhood of $\tilde{A}$ to make the image of $\tilde{A}$ disjoint from $E$. This modification can be performed equivariantly on each component of the $\pi_{1}(M)$-orbit of $\tilde{A}$. This produces a new equivariant map $\tilde{\phi}_{1}: \tilde{M} \rightarrow T_{F}$. As in [CGLS] the map $\tilde{\phi}_{1}$ determines a surface $S_{1}$ which is also associated to the action of $\pi_{1}(M)$ on $T_{F}$ and which differs from $S$ by a surgery on the annulus $A$, i.e $S_{1}$ is obtained from $S$ by "tubing" along the annulus $A$. This is a contradiction since $S_{1}$ has fewer boundary components than $S$, which proves the claim.

For the proof of the proposition, let $C=C_{0}, \ldots, C_{n}=C^{\prime}$ be the components of $\partial \tilde{S}$ which lie between $C$ and $C^{\prime}$ on $U$, numbered consecutively. There is a unique edge path $e_{0}, \ldots, e_{n}$ such that for each $i=0, \ldots, n$ either $e_{i}=e\left(C_{i}\right)$ or $e_{i}=\overline{e\left(C_{i}\right)}$; specifically we have $e_{i}=e\left(C_{i}\right)$ if the transverse orientation on $C_{i}$ points toward $C_{i+1}$ and $e_{i}=\overline{e\left(C_{i}\right)}$ otherwise. The claim proved above implies that $e_{i} \neq \bar{e}_{i+1}$ irrespective of whether the orientations of $C_{i}$ and $C_{i+1}$ are compatible. Therefore this edge path is reduced, so $e_{0}$ and $e_{n}$ are comparable, as are $\bar{e}_{0}$ and $\bar{e}_{n}$. The orientations on $C$ and $C^{\prime}$ are compatible if and only if either $e_{0}=e(C)$ and $e_{n}=e\left(C^{\prime}\right)$ or $\bar{e}_{0}=e(C)$ and $\bar{e}_{n}=e\left(C^{\prime}\right)$. Thus $e(C)$ is comparable to $e\left(C^{\prime}\right)$ if and only if the orientations on $C$ and $C^{\prime}$ are compatible.

5.7. We continue to let $M$ denote a compact orientable 3 -manifold whose boundary is a torus, and $P$ a representation of $\pi_{1}(M)$ in $\mathrm{SL}_{2}(F)$, where $F$ is a field with a discrete valuation. Note that $P$ induces a representation of $\pi_{1}(\partial M)$ in $\mathrm{SL}_{2}(F)$ which is defined up to conjugacy. The image of this representation will be denoted $P\left(\pi_{1}(\partial M)\right)$. 
If $S$ is an essential surface in $M$ then since the boundary components of $S$ are parallel simple closed curves on $\partial M$ they lie in the same unoriented free homotopy class. Thus they determine a unique pair $\left[h^{ \pm 1}\right]$ of conjugacy classes in $\pi_{1}(M)$. The trace of an element of $\mathrm{SL}_{2}(F)$ is unchanged by conjugation and taking inverses, so we may define $\operatorname{tr} \partial S=\operatorname{tr} P(h)$.

If $S$ is a compact 2-dimensional manifold with non-empty boundary then $n(S)$ denotes the greatest common divisor of the numbers of boundary components of the various components of $S$.

Theorem. Suppose that $P\left(\pi_{1}(\partial M)\right)$ is not conjugate to a subgroup of $\mathrm{SL}_{2}(\mathcal{O})$. Then $\operatorname{tr} \partial S=\lambda+\lambda^{-1}$ where $\lambda$ is a root of unity. Moreover, if $S$ is a reduced surface associated to the action of $\pi_{1}(M)$ on $T_{F}$ determined by $P$ then $\lambda^{n(S)}=1$.

Proof. As in Section 4, we fix orientations of $S$ and $M$. We consider an arbitrary component $S_{0}$ of $S$ with $\partial S_{0} \neq \emptyset$. Choose a component $\tilde{S}_{0}$ of $p^{-1}\left(S_{0}\right)$. Then $\tilde{S}_{0}$ is a simply connected cover of $S_{0}$; we write $\pi_{1}\left(S_{0}\right)$ for its group of deck transformations, which is the stabilizer of $\tilde{S}_{0}$ in $\pi_{1}(M)$. The image of $\tilde{S}_{0}$ is the midpoint of an edge $e$ where the direction of $e$ pulls back to the transverse orientation of $S_{0}$.

By equivariance, the stabilizer of $\tilde{S}_{0}$ is contained in the stabilizer of $e$. A component $C$ of $\partial S$, with its induced orientation, determines a conjugacy class in the stabilizer of $\tilde{S}_{0}$, which in turn determines a conjugacy class in the stabilizer of $e$. Choose a representative $h$ of the latter conjugacy class and define $\lambda_{C}$ to be the eigenvalue of $h$ associated to $e$. By the remarks at the end of Section $2, \lambda_{C}$ is independent of the choice of $h$. Since $\pi_{1}(M)$ acts transitively on the components of $p^{-1}\left(S_{0}\right), \lambda_{C}$ is also independent of the choice of $\tilde{S}_{0}$.

We claim that if $C$ and $C^{\prime}$ are two components of $\partial S_{0}$ then $\lambda_{C}=\lambda_{C^{\prime}}$. To prove this we consider an arbitrary component $U$ of $\partial \tilde{M}$. Let $\tilde{C}$ and $\tilde{C}^{\prime}$ be components of $p^{-1}(C)$ and $p^{-1}\left(C^{\prime}\right)$ contained in $U$. Let $\tilde{S}_{0}$ and $\tilde{S}_{0}^{\prime}$ denote the components of $p^{-1}\left(S_{0}\right)$ containing $\tilde{C}$ and $\tilde{C}^{\prime}$ respectively. Then $\tilde{\phi}\left(\tilde{S}_{0}\right)$ and $\tilde{\phi}\left(\tilde{S}_{0}^{\prime}\right)$ are midpoints of edges $e$ and $e^{\prime}$ whose directions pull back to the transverse orientations of $\tilde{S}_{0}$ and $\tilde{S}_{0}^{\prime}$. Let $h$ be the generator of the stabilizer of $\tilde{C}$ corresponding to the oriented curve $C$. Now $h$ is a deck transformation of the covering of the torus $\partial M$ by $U$ and leaves $\tilde{C}$ invariant. Since $h$ also preserves the entire family $U \cap p^{-1}(\partial S)$ of parallel lines or circles, it must leave each member of the family invariant. In particular, $C^{\prime}$ is invariant under $h$. However, $h$ will be the generator of the stabilizer of $\tilde{C}^{\prime}$ corresponding to the oriented curve $C^{\prime}$ if and only if the orientations of $\tilde{C}$ and $\tilde{C}^{\prime}$ agree. Otherwise $h^{-1}$ will be this generator.

By Proposition 5.6 the edges $e=e(\tilde{C})$ and $e^{\prime}=e\left(\tilde{C}^{\prime}\right)$ are comparable if and only if the orientations of $\tilde{C}$ and $\tilde{C}^{\prime}$ are compatible. The eigenvalue of $h$ associated to $e$ is $\lambda_{C}$. If the orientations of $\tilde{C}$ and $\tilde{C}^{\prime}$ are compatible then $\lambda_{C^{\prime}}$ is the eigenvalue of $h$ associated to $e^{\prime}$; by Proposition $5.3 \lambda_{C}=\lambda_{C^{\prime}}$ since $e$ is comparable to $e^{\prime}$. If the orientations are not compatible then $\lambda_{C^{\prime}}$ is the eigenvalue of $h^{-1}$ associated to $e^{\prime}$ which, by Proposition 5.2, 
equals the eigenvalue of $g$ associated to $\overline{e^{\prime}}$. By Proposition 5.3 this equals the eigenvalue of $g$ associated to $e$ since $e$ and $e^{\prime}$ are not comparable. Thus we again have $\lambda_{C}=\lambda_{C^{\prime}}$.

To complete the proof of the theorem we let the boundary components of $S_{0}$ be $C_{0}, \ldots, C_{m}$. For $i=0, \ldots, m$ let $h_{i}$ be an element of $\pi_{1}\left(S_{0}\right)$ in the conjugacy class determined by $C_{i}$ with the induced orientation. Then each $h_{i}$ is an element of the stabilizer of $e$ and the eigenvalue of $h_{i}$ associated to the edge $e$ is $\lambda_{i}=\lambda_{C_{i}}$. Moreover, the product $h_{0} \ldots h_{m}$ is an element of the commutator subgroup of $\pi_{1}\left(S_{0}\right)$ and hence of the commutator subgroup of the stabilizer of $e$. Now, the commutator subgroup of the stabilizer of an edge of $T_{F}$ consists of unipotent elements $\bmod \pi$. Thus we have $\lambda_{1} \ldots \lambda_{m}=1 \bmod \pi$. By the claim proved above $\lambda_{1}=\ldots=\lambda_{m}$. If we let $\lambda$ be the image of $\lambda_{i}$ in the residue field $k$ then we have $\operatorname{tr} \partial S=\lambda+\lambda^{-1}$ where $\lambda^{m}=1$. Since $S_{0}$ was an arbitrary component of $S$, this implies the statement of the theorem.

Corollary. Let $M$ be a compact orientable 3-manifold with boundary a torus. Let $x$ be an ideal point of $D_{M}$. Let $Y$ be the component of $D_{M}$ and $v$ the valuation on $\mathbf{C}(Y)$ associated to $x$. If the incompressible surfaces associated to $x$ have boundary slope $p / q$, where $(p, q)=1$, then the field value $\tau_{v}(\mathrm{l}, \mathrm{m})$ of $\mathrm{m}^{p} / \mathrm{l}^{q}$ at $x$ is a root of unity. If $S$ is any component of a reduced surface associated to the ideal point $z$ then $\tau_{v}(l, m)^{n(S)}=1$.

ProOf. Let $Y$ be the irreducible component of $D_{M}$ such that $x$ is an ideal point of $Y$. Let $F$ be the finite extension of $\mathbf{C}(Y)$ provided by Proposition 2.2 equipped with a discrete valuation which extends the valuation on $\mathbf{C}(Y)$ associated to $x$. Apply the Theorem to $P$.

5.8. The K-theoretic argument given in section 4 implies that the order of $\lambda$ divides $2 N \operatorname{gcd}\left(v^{\prime}(\mathrm{l}), v^{\prime}(\mathrm{m})\right)$ where $v^{\prime}$ is an extension of $v$ to a valuation on $F$ and satisfies $v^{\prime}(f)=$ $N v(f)$ for $f \in \mathbf{C}(Y)$. Interpreted in terms of the tree this shows that the order of $\lambda$ divides the number of boundary components of any reduced surface associated to $z$. Thus Corollary 5.7 gives stronger information when this surface is not connected.

5.9. Corollary 5.7 gives insight into the proof of the Smith Conjecture. In [CS1] Culler and Shalen proved a strong version of the Smith Conjecture: If $\tilde{\Sigma}$ is an $n$-fold cyclic cover $(n>1)$ of a closed 3-manifold $\Sigma$ branched over a non-trivial knot $K$ then either

(1) $\tilde{\Sigma}$ contains a non-separating 2 -sphere or an incompressible surface of positive genus; or

(2) $\pi_{1}(\tilde{\Sigma})$ admits a non-trivial representation in $\operatorname{PSL}_{2}(\mathbf{C})$.

In the case where $\Sigma-K$ is hyperbolic this was proved by considering a suitable curve $X$ in the character variety of $\pi_{1}(\Sigma-K)$, and the function $I_{M}$ where $M$ is a meridian of $K$. Let 
$\hat{X}$ denote the smooth projective model of $X$ and $\hat{I}_{M}$ the extension of $I_{M}$ to a function on $\hat{X}$. There always exists a point $x \in \hat{X}$ such that $\hat{I}_{\mathrm{m}}(x)=\zeta+\zeta^{-1}$, where $\lambda$ is a primitive $n^{\text {th }}$ root of unity. If $x$ is an ideal point then conclusion (1) holds; if $x \in X$ then conclusion (2) holds.

It would appear that this proof involves only rather special ideal points, namely those where some $\hat{I}_{g}$, for $g \in \pi_{1}(\partial M)$, takes a value of the form $\zeta+\zeta^{-1}$ with $\zeta$ a root of unity. Corollary 5.7 says that these ideal points are not so special.

In the proof of the Cyclic Surgery Theorem [CGLS], an important rôle is played by ideal points where some $\hat{I}_{g}$ takes the value 2 or -2 . These correspond to the cases where $\zeta= \pm 1$ in the conclusion of Corollary 5.7. The statement of 5.7 leaves open the possibility that $\zeta$ is always \pm 1 . It would be very interesting to give an example for which $\zeta \neq \pm 1$.

5.10. We record here the conditions which are imposed upon the coefficients of $A_{M}$ by the conclusion of Corollary 5.7. Assume that

$$
A_{M}(\mathrm{l}, \mathrm{m})=\sum b_{m n} \mathrm{l}^{m} \mathrm{~m}^{n} .
$$

Proposition. Suppose that the Newton polygon of $A_{M}$ has a side of slope $-p / q$ and let $E$ be the set of lattice points $(m, n)$ which lie on this side. Then the polynomial

$$
\Theta(z)=\sum_{(m, n) \in E} b_{m n} z^{n}
$$

is a product of cyclotomic polynomials. The orders of the roots of unity which occur as roots of $\Theta_{M}$ divide the number of boundary components of any component of a reduced surface determined by the given side of the Newton polygon.

\section{§6. Alexander Polynomials.}

In the case where $M$ is the complement of a knot $K$ in a homology 3 -sphere, the polynomial $A_{K}$ is related to the Alexander polynomial of $K$. This relationship will be described here. We will write $\Delta_{K}(t)$ for the Alexander polynomial of $K$. Recall that the $\Delta_{K}$ is a Laurent polynomial which is well defined up to multiplication by units. 
6.1. It was observed by de Rham $[\mathrm{dR}]$ that the Alexander polynomial of $K$ can be regarded as the defining equation of a curve of representations of $\pi_{1}(M)$ into the affine group of the complex plane. In de Rham's formulation one considers meridian generators $g_{0}, g_{1}, \ldots, g_{n}$ of the knot group and a representation $\alpha$ into the affine group which does not have abelian image. Assume that $\alpha$ has been normalized up to conjugation in the affine group so that the fixed point of $\alpha\left(g_{0}\right)$ is 0 . Thus $\alpha\left(g_{0}\right)$ is the affine transformation $z \rightarrow t z$ for some complex number $t$. Since the meridians are all conjugate elements of $\pi_{1}(M)$, the affine transformations $\alpha\left(g_{i}\right)$ all have the same derivative. In particular there exist complex numbers $x_{1}, \ldots, x_{n}$ so that $\alpha\left(g_{i}\right)$ is the affine transformation $z \rightarrow t z+x_{n}$. The condition that $\alpha$ have non-abelian image is equivalent to the condition that some $x_{i}$ be non-zero. Setting the image of a relator in $\pi_{1}(M)$ equal to 1 gives rise to a homogeneous linear equation in $x_{1}, \ldots, x_{n}$ whose coefficients are polynomials in $t$. An n-relator presentation of $\pi_{1}(M)$, e.g. a Wirtinger presentation, determines a homogeneous system of linear equations over the polynomial ring $\mathbf{C}[t]$. In $[\mathrm{dR}]$ it is shown that the determininant of this system is well-defined up to multiplication by powers of $t$ and is the Alexander polynomial of $K$. Thus if $\alpha$ is any affine representation of $\pi_{1}(M)$ then there is a root $t$ of the Alexander polynomial such that the derivative of $\rho(g)$ equals $t$ for any meridian $g$. Conversely, if $\alpha$ is a function sending the generators $g_{0} \ldots g_{n}$ to the affine transformations given above, then $\alpha$ extends to a representation with non-abelian image if and only if the linear system has a non-zero solution. Therefore there exists a non-abelian affine representation of $\pi_{1}(M)$ for which the image of each meridian has derivative $t$ if and only if $\Delta_{K}(t)=0$.

Lemma. Let $K$ be a knot in a homology 3-sphere $\Sigma$. Let $M$ be the complement of $K$ in $\Sigma$. Then any representation of $\pi_{1}(M)$ into $\mathrm{PSL}_{2}(\mathbf{C})$ lifts to a representation of $\pi_{1}(M)$ into $\mathrm{SL}_{2}(\mathbf{C})$.

Proof. First assume that $M$ is irreducible. To prove the lemma it suffices to show that any central extension of the cyclic group of order 2 by $\pi_{1}(M)$ is split. Since $M$ is a $K(\pi, 1)$-space, this is equivalent to the statement $H^{2}\left(M, \mathbf{Z}_{2}\right)=0$, which is immediate since $M$ is a homology $S^{1}$. In the general case $M$ is a connected sum of irreducible manifolds one of which is a homology $S^{1}$ while the others are homology 3-spheres. A representation of a free product lifts if and only if the restrictions to the factors lift. $\mathrm{A} \mathrm{PSL}_{2}(\mathbf{C})$ representation of the fundamental group of an irreducible summand of $M$ lifts to $\mathrm{SL}_{2}(\mathbf{C})$ again because its second cohomology group with $\mathbf{Z}_{2}$ coefficients vanishes.

Proposition. Let $M$ be the complement of a knot $K$ in a homology 3-sphere. The following are equivalent.

1. There exists a reducible representation of $\pi_{1}(M)$ in $\mathrm{SL}_{2}(\mathbf{C})$ which has non-abelian image and sends $\mathrm{M}$ to an element with eigenvalue $\mathrm{m}$. 
2. $\mathrm{m}^{2}$ is a root of $\Delta_{K}$.

Proof. This is immediate from the lemma and the preceding discussion.

6.2. Proposition. Let $M$ be the complement of a knot $K$ in a homology 3-sphere. Suppose that $\rho$ is a reducible representation of $\pi_{1}(M)$ such that the character of $\rho$ lies on a component of $X^{\prime}$ which contains the character of an irreducible representation. Then $\rho(\mathrm{M})$ has eigenvalue $\mathrm{m}$ where $\mathrm{m}^{2}$ is a root of $\Delta_{K}$.

ProOF. We need only show that there is a representation with the same character as $\rho$ which has non-abelian image. It is shown in [CS1] that if $Y$ is a component of $X$ which contains the character of an irreducible representation then $t^{-1}(\chi)$ is 3 -dimensional for each $\chi \in Y$. However, by [CGLS] the variety of abelian representations with a given character is 2-dimensional. Thus $t^{-1}\left(\chi_{\rho}\right)$ contains a representation with non-abelian image.

6.3. Recall that the longitude of a knot is an element of the second derived subgroup of the knot group. This means that if $M$ is the complement of a knot in a homology 3-sphere then $\mathrm{L}$ is in the kernel of any reducible representation of $\pi_{1}(M)$ in $\mathrm{SL}_{2}(\mathbf{C})$. In particular, if $\rho$ is a reducible representation of $\pi_{1}(M)$ such that $\chi_{\rho} \in X^{\prime}$ then the corresponding point of $D_{K}$ will have l-coordinate 1 . If $\chi_{\rho}$ lies on a component of $X^{\prime}$ which contains the character of an irreducible representation then Proposition 6.2 shows that the $y$-coordinate of this point of $D_{K}$ is $\mathrm{m}$ where $\Delta\left(\mathrm{m}^{2}\right)=0$. We remarked in Section 2 that the component of $D_{M}$ which corresponds to a component of $X^{\prime}$ consisting entirely of reducible characters is just the line $l=1$. Thus we have the following.

Proposition. Let $V$ be an irreducible component of $D_{K}$ distinct from the line $l=1$. The points of $V$ which correspond to reducible characters have coordinates $(1, t)$ where $\Delta\left(t^{2}\right)=0$. In this case the minimal polynomial of $t$ is a common irreducible factor of $A_{K}(1, \mathrm{~m})$ and $\Delta_{K}\left(\mathrm{~m}^{2}\right)$. 


\section{§. Computations}

The computation of $A_{M}$ can be reduced to classical elimination theory and is therefore, in principle, possible for arbitrary $M$. Suppose that we are given a representation $\rho: \pi_{1}(M) \rightarrow$ $\mathrm{SL}_{2}(\mathbf{C})$ which restricts to an upper triangular representation of the peripheral subgroup of $\pi_{1}(M)$. A diagonal representation $\rho^{\prime}$ of $\pi_{1}(\partial M)$ can be constructed as follows:

$$
\text { If } \quad \rho(\gamma)=\left[\begin{array}{cc}
a(\gamma) & b(\gamma) \\
0 & d(\gamma)
\end{array}\right] \text { then } \quad \rho^{\prime}(\gamma)=\left[\begin{array}{cc}
a(\gamma) & 0 \\
0 & d(\gamma)
\end{array}\right] \text {. }
$$

It follows from the definition that $\rho^{\prime}$ is a point of $D_{M} \subset \mathbf{C}^{*} \times \mathbf{C}^{*}$ with coordinates $(a(\mathrm{~L}), a(\mathrm{M}))$. Since any representation in $R(M)$ is conjugate to a representation which is upper triangular on the peripheral subgroup, there is a dense subset of $D_{M}$ which is produced by this construction.

A finite presentation of $\pi_{1}(M)$ determines an explicit set of defining equations for the affine variety $R(M)$. The affine coordinates of a representation $\rho$ are the matrix entries of the images of the generators. Each relator determines a matrix whose entries are polynomials in these coordinates; setting these matrices equal to the identity produces a set of defining equations. Suppose that the elements $L$ and $M$ are included in the generating set of $\pi_{1}(M)$. Let $U$ be the subvariety of $R(M)$ consisting of representations $\rho$ with $\rho(\mathrm{L})$ and $\rho(\mathrm{M})$ upper triangular. Then $D_{M}$ is the closure of the planar projection of $U$ defined by the two coordinates $a(\mathrm{~L})$ and $a(\mathrm{M})$.

These computations are especially tractable in the case of 2-bridge knots. In this case the usual presentation of $\pi_{1}(M)$ has the form

$$
\langle x, y: x w=w y\rangle
$$

where $x$ and $y$ are meridians. We set $\mathrm{M}=x$ and $\mathrm{L}=x^{n} w w^{*}$ where $w^{*}$ is the word obtained by reversing $w$ and $n$ is chosen to make the exponent sum of $\mathrm{L}$ be 0 . It suffices to compute a defining equation $A^{\prime}(\mathrm{l}, \mathrm{m})$ for the curve $D_{M}^{\prime}$ which is the union of the irreducible components of $D_{M}$ other than the line $l=1$. (This line contains the points of $D_{M}$ which are determined by reducible representations in $U$.) Suppose that $\rho$ is an irreducible representation in $U$. After conjugation and after replacing $y$ by $y^{-1}$ if necessary we can assume that

$$
\rho(x)=\left[\begin{array}{cc}
\mathrm{m} & 1 \\
0 & \mathrm{~m}^{-1}
\end{array}\right] \quad \text { and } \quad \rho(y)=\left[\begin{array}{cc}
\mathrm{m} & 0 \\
t & \mathrm{~m}^{-1}
\end{array}\right] .
$$

Let $p(\mathrm{~m}, t)$ be the upper right entry of the matrix $\rho(x w)-\rho(w y)$, which has diagonal entries equal to 0 . Let $q(\mathrm{~m}, t)$ be the upper left entry of $\rho\left(x^{n} w w^{*}\right)$. The curve $D_{M}^{\prime}$ is the closure of the projection onto the $(\mathrm{l}, \mathrm{m})$ plane of the curve defined by $p=0$ and $q=\mathrm{l}$. Thus 
$A^{\prime}(l, \mathfrak{m})$ is obtained by deleting repeated factors from the resultant of $\mathrm{m}^{r} p$ and $\mathrm{m}^{s}(q-l)$ over $t$, where the exponents $r$ and $s$ are chosen to make $\mathrm{m}^{r} p$ and $\mathrm{m}^{s}(q-l)$ be polynomials.

The appendix to this paper lists the polynomials $A_{K}$ for a number of knots, including some which are not 2-bridge knots. In order to make extraction of the Newton polygon as easy as possible, the polynomials are specified by giving matrices of coefficients. An $m \times n$ matrix $Q$ represents the polynomial

$$
\left[\begin{array}{llll}
1 & \mathrm{~m}^{2} & \cdots & \mathrm{m}^{2 m-2}
\end{array}\right] Q\left[\begin{array}{llll}
1 & \mathrm{l} & \cdots & \mathrm{l}^{n-1}
\end{array}\right]^{\mathrm{T}} .
$$

One computation which is not included in the appendix, because of the enormous size of the polynomial, is that of $A_{K}$ where $K$ is the untwisted double of the trefoil.

As an indication of the kind of topological information that can be obtained from these computations we mention that the results in the appendix provide evidence for the following conjecture, which implies that if a non-trivial knot has no essential closed surface in its complement then no non-trivial Dehn surgery on the knot produces a simply-connected manifold.

Conjecture. For any non-trivial knot $K$ the degree of $A_{K}$ as a polynomial in $\mathrm{m}$ is more than twice its degree as a polynomial in $\mathrm{l}$.

To make the connection with Dehn surgery, note that the conjecture above implies that the degree of $I_{\mathrm{L}}$, as a function on the character variety of the complement $M$ of $K$, is at least twice that of $I_{M}$. We know from [CGLS] that

(i) there is a norm $|\cdot|$ on the vector space $\mathbf{R}^{2}$ such that if $p$ and $q$ are integers then $|(p, q)|$ is the degree of $I_{\gamma}$ for $\gamma=\mathrm{L}^{p} \mathrm{M}^{q}$; and

(ii) if $p / q$-surgery on $K$ produces a manifold with cyclic fundamental group then either $p / q$ is the boundary slope of an incompressible surface in $M$ or the vector $(p, q)$ has minimal norm among non-zero vectors with integer coordinates.

Suppose that $p / q$-surgery on $K$ produces a simply-connected manifold. By the Cyclic Surgery Theorem of [CGLS], we know that $p / q= \pm 1$. If neither $1 / 0$ nor $p / q$ are boundary slopes for $K$ then then we would have $|(1,0)|=|(1, \pm 1)|$. By the triangle inequality this implies $|(0,1)| \leq 2|(1,0)|$, contradicting the conjecture above. On the other hand, it is shown in [CGLS] that if $M$ contains no essential closed surface then surgery along a boundary slope cannot produce a simply-connected manifold.

In the general case the conjecture would reduce the question of whether non-trivial surgery can produce a simply connected manifold to the following question, which has the flavor of the results in the second chapter of [CGLS]. If a surgery on a boundary slope produces a simply-connected manifold, must every other surgery produce a non-simplyconnected manifold? 


\section{References}

[CS1] M. Culler and P. B. Shalen, "Varieties of group reprsentations and splittings of 3-manifolds," Annals of Math., 117(1983), 109-146.

[CS2] M. Culler and P. B. Shalen, "Bounded, separating, incompressible surfaces in knot manifolds," Invent. math., 75(1984), 537-545.

[CGLS] M. Culler, C. McA. Gordon, J. Luecke and P. B. Shalen, "Dehn surgery on knots," Annals of Math., 125(1987), 237-300.

[H] A. E. Hatcher, "On the boundary curves of incompressible surfaces," Pac. J. Math., 99(1982), 373-377.

[K] F. Kirwan, Complex Algebraic Curves, Londan Math. Soc. Student Texts 23, Cambridge University Press, Cambridge, 1992.

[La] S. Lang, Introduction to Algebraic Geometry, Interscience Publishers, New York, 1958.

[Le] S. Lefshetz, Algebraic Geometry, Princeton University Press, Princeton, 1953.

[R] D. Ramakrishnan, "Regulators, Algebraic Cycles, and Values of L-Functions," Contemporary Mathematics, 83(1989), 183-310.

[H] C. Hodgson, "Degeneration and Regeneration of Hyperbolic Structures on Three-Manifolds," Thesis, Princeton University, 1986.

[M] J. Milnor, Introduction to Algebraic K-theory, Annals of Math. Study no. 72, Princeton University Press, Princeton, 1971.

[S] J.-P. Serre (with H. Bass), Trees, Springer-Verlag, 1980. 


\section{Appendix}

$3_{1}$

$\left(\begin{array}{ll}0 & 1 \\ 0 & 0 \\ 0 & 0 \\ 1 & 0\end{array}\right)$

$4_{1}$

$\left(\begin{array}{rrr}0 & 1 & 0 \\ 0 & -1 & 0 \\ -1 & -2 & -1 \\ 0 & -1 & 0 \\ 0 & 1 & 0\end{array}\right)$

$\left(\begin{array}{ll}0 & 1 \\ 0 & 0 \\ 0 & 0 \\ 0 & 0 \\ 0 & 0 \\ 1 & 0\end{array}\right)$

$\left(\begin{array}{rrrr}1 & -1 & 0 & 0 \\ 0 & 2 & 0 & 0 \\ 0 & 2 & 1 & 0 \\ 0 & 0 & -1 & 0 \\ 0 & -1 & 0 & 0 \\ 0 & 1 & 2 & 0 \\ 0 & 0 & 2 & 0 \\ 0 & 0 & -1 & 1\end{array}\right)$

$6_{1}$

$$
\left(\begin{array}{rrrrr}
0 & 1 & -1 & 0 & 0 \\
0 & -1 & 3 & 0 & 0 \\
0 & 0 & 1 & 2 & 0 \\
0 & 0 & -3 & -3 & 0 \\
-1 & -3 & -6 & -3 & -1 \\
0 & -3 & -3 & 0 & 0 \\
0 & 2 & 1 & 0 & 0 \\
0 & 0 & 3 & -1 & 0 \\
0 & 0 & -1 & 1 & 0
\end{array}\right)
$$

$$
\left(\begin{array}{rrrrrr}
0 & 0 & 0 & 0 & 1 & 0 \\
0 & 0 & 0 & 1 & -2 & 0 \\
0 & 0 & 0 & -3 & 1 & -1 \\
0 & 0 & 0 & 1 & 2 & 0 \\
0 & 0 & 0 & 5 & -5 & 0 \\
0 & 0 & -3 & 3 & -5 & 0 \\
0 & 0 & 8 & -12 & 3 & 0 \\
0 & 0 & 3 & -13 & 0 & 0 \\
0 & 0 & -13 & 3 & 0 & 0 \\
0 & 3 & -12 & 8 & 0 & 0 \\
0 & -5 & 3 & -3 & 0 & 0 \\
0 & -5 & 5 & 0 & 0 & 0 \\
0 & 2 & 1 & 0 & 0 & 0 \\
-1 & 1 & -3 & 0 & 0 & 0 \\
0 & -2 & 1 & 0 & 0 & 0 \\
0 & 1 & 0 & 0 & 0 & 0
\end{array}\right)
$$


$6_{3}$

$$
\left(\begin{array}{rrrrrrr}
0 & 0 & 0 & 1 & 0 & 0 & 0 \\
0 & 0 & 1 & -5 & 1 & 0 & 0 \\
0 & 0 & -4 & 3 & -4 & 0 & 0 \\
0 & 0 & 4 & 9 & 4 & 0 & 0 \\
0 & 2 & 2 & -2 & 2 & 2 & 0 \\
0 & -5 & -6 & -21 & -6 & -5 & 0 \\
0 & 1 & 2 & 8 & 2 & 1 & 0 \\
1 & 10 & 17 & 34 & 17 & 10 & 1 \\
0 & 1 & 2 & 8 & 2 & 1 & 0 \\
0 & -5 & -6 & -21 & -6 & -5 & 0 \\
0 & 2 & 2 & -2 & 2 & 2 & 0 \\
0 & 0 & 4 & 9 & 4 & 0 & 0 \\
0 & 0 & -4 & 3 & -4 & 0 & 0 \\
0 & 0 & 1 & -5 & 1 & 0 & 0 \\
0 & 0 & 0 & 1 & 0 & 0 & 0
\end{array}\right)
$$

$7_{1}$

$$
\left(\begin{array}{ll}
1 & 0 \\
0 & 0 \\
0 & 0 \\
0 & 0 \\
0 & 0 \\
0 & 0 \\
0 & 0 \\
0 & 1
\end{array}\right)
$$

$$
\left(\begin{array}{rrrrrr}
0 & 0 & 0 & 1 & -2 & 1 \\
0 & 0 & 0 & -4 & 4 & 0 \\
0 & 0 & -2 & 2 & 3 & 0 \\
0 & 0 & 5 & 5 & 0 & 0 \\
0 & 1 & 1 & 6 & 0 & 0 \\
0 & -1 & -4 & 0 & 0 & 0 \\
0 & 0 & 0 & -4 & -1 & 0 \\
0 & 0 & 6 & 1 & 1 & 0 \\
0 & 0 & 5 & 5 & 0 & 0 \\
0 & 3 & 2 & -2 & 0 & 0 \\
0 & 4 & -4 & 0 & 0 & 0 \\
1 & -2 & 1 & 0 & 0 & 0
\end{array}\right)
$$




\section{$7_{3}$}

$\left(\begin{array}{rrrrrrr}1 & -1 & 0 & 0 & 0 & 0 & 0 \\ 0 & 2 & 0 & 0 & 0 & 0 & 0 \\ 0 & -1 & 0 & 0 & 0 & 0 & 0 \\ 0 & 0 & 0 & 0 & 0 & 0 & 0 \\ 0 & 5 & 3 & 0 & 0 & 0 & 0 \\ 0 & 2 & -9 & 0 & 0 & 0 & 0 \\ 0 & -3 & 2 & 0 & 0 & 0 & 0 \\ 0 & 2 & 14 & 0 & 0 & 0 & 0 \\ 0 & 0 & 2 & -3 & 0 & 0 & 0 \\ 0 & 0 & -4 & 10 & 0 & 0 & 0 \\ 0 & 0 & 4 & -3 & 0 & 0 & 0 \\ 0 & 0 & 2 & -12 & 0 & 0 & 0 \\ 0 & 0 & 3 & 6 & 1 & 0 & 0 \\ 0 & 0 & -3 & 24 & -3 & 0 & 0 \\ 0 & 0 & 1 & 6 & 3 & 0 & 0 \\ 0 & 0 & 0 & -12 & 2 & 0 & 0 \\ 0 & 0 & 0 & -3 & 4 & 0 & 0 \\ 0 & 0 & 0 & 10 & -4 & 0 & 0 \\ 0 & 0 & 0 & -3 & 2 & 0 & 0 \\ 0 & 0 & 0 & 0 & 14 & 2 & 0 \\ 0 & 0 & 0 & 0 & 2 & -3 & 0 \\ 0 & 0 & 0 & 0 & -9 & 2 & 0 \\ 0 & 0 & 0 & 0 & 3 & 5 & 0 \\ 0 & 0 & 0 & 0 & 0 & 0 & 0 \\ 0 & 0 & 0 & 0 & 0 & -1 & 0 \\ 0 & 0 & 0 & 0 & 0 & 2 & 0 \\ 0 & 0 & 0 & 0 & 0 & -1 & 1\end{array}\right)$

$7_{4}$

$$
\left(\begin{array}{rrrrrr}
1 & -3 & 3 & -1 & 0 & 0 \\
0 & 7 & -10 & 3 & 0 & 0 \\
0 & 4 & 3 & -3 & 0 & 0 \\
0 & -6 & 21 & -2 & 0 & 0 \\
0 & 1 & -3 & 10 & 1 & 0 \\
0 & 3 & -17 & 6 & -2 & 0 \\
0 & -2 & 6 & -17 & 3 & 0 \\
0 & 1 & 10 & -3 & 1 & 0 \\
0 & 0 & -2 & 21 & -6 & 0 \\
0 & 0 & -3 & 3 & 4 & 0 \\
0 & 0 & 3 & -10 & 7 & 0 \\
0 & 0 & -1 & 3 & -3 & 1
\end{array}\right)
$$




$\left(\begin{array}{rrrrrrrrr}0 & 0 & 0 & 0 & 0 & 0 & 0 & 1 & -1 \\ 0 & 0 & 0 & 0 & 0 & 0 & 0 & -4 & 0 \\ 0 & 0 & 0 & 0 & 0 & 0 & -1 & 5 & 0 \\ 0 & 0 & 0 & 0 & 0 & 0 & 6 & 2 & 0 \\ 0 & 0 & 0 & 0 & 0 & 0 & -17 & -13 & 0 \\ 0 & 0 & 0 & 0 & 0 & -2 & 10 & -3 & 0 \\ 0 & 0 & 0 & 0 & 0 & 12 & 35 & 7 & 0 \\ 0 & 0 & 0 & 0 & 0 & -23 & -32 & -3 & 0 \\ 0 & 0 & 0 & 0 & -1 & -6 & -56 & 0 & 0 \\ 0 & 0 & 0 & 0 & 6 & 48 & 24 & 0 & 0 \\ 0 & 0 & 0 & 0 & -11 & 15 & 28 & 0 & 0 \\ 0 & 0 & 0 & 0 & 4 & -82 & -22 & 0 & 0 \\ 0 & 0 & 0 & 0 & 4 & -28 & -14 & 0 & 0 \\ 0 & 0 & 0 & -1 & 4 & 47 & 14 & 0 & 0 \\ 0 & 0 & 0 & 7 & -11 & -13 & -3 & 0 & 0 \\ 0 & 0 & 0 & -12 & 12 & -46 & 0 & 0 & 0 \\ 0 & 0 & 0 & -2 & -16 & 15 & 0 & 0 & 0 \\ 0 & 0 & 0 & 15 & -52 & 15 & 0 & 0 & 0 \\ 0 & 0 & 0 & 15 & -16 & -2 & 0 & 0 & 0 \\ 0 & 0 & 0 & -46 & 12 & -12 & 0 & 0 & 0 \\ 0 & 0 & -3 & -13 & -11 & 7 & 0 & 0 & 0 \\ 0 & 0 & 14 & 47 & 4 & -1 & 0 & 0 & 0 \\ 0 & 0 & -14 & -28 & 4 & 0 & 0 & 0 & 0 \\ 0 & 0 & -22 & -82 & 4 & 0 & 0 & 0 & 0 \\ 0 & 0 & 28 & 15 & -11 & 0 & 0 & 0 & 0 \\ 0 & 0 & 24 & 48 & 6 & 0 & 0 & 0 & 0 \\ 0 & 0 & -56 & -6 & -1 & 0 & 0 & 0 & 0 \\ 0 & -3 & -32 & -23 & 0 & 0 & 0 & 0 & 0 \\ 0 & 7 & 35 & 12 & 0 & 0 & 0 & 0 & 0 \\ 0 & -3 & 10 & -2 & 0 & 0 & 0 & 0 & 0 \\ 0 & -13 & -17 & 0 & 0 & 0 & 0 & 0 & 0 \\ 0 & 2 & 6 & 0 & 0 & 0 & 0 & 0 & 0 \\ 0 & 5 & -1 & 0 & 0 & 0 & 0 & 0 & 0 \\ 0 & -4 & 0 & 0 & 0 & 0 & 0 & 0 & 0 \\ -1 & 1 & 0 & 0 & 0 & 0 & 0 & 0 & 0\end{array}\right)$


$7_{6}$

$$
\left(\begin{array}{rrrrrrrrrr}
0 & 0 & 0 & 0 & 0 & 0 & 1 & -1 & 0 & 0 \\
0 & 0 & 0 & 0 & 0 & 0 & -7 & 6 & 0 & 0 \\
0 & 0 & 0 & 0 & 0 & -1 & 16 & -11 & 2 & 0 \\
0 & 0 & 0 & 0 & 0 & 9 & -1 & -2 & -6 & 0 \\
0 & 0 & 0 & 0 & 0 & -32 & -34 & 16 & 5 & -1 \\
0 & 0 & 0 & 0 & -2 & 30 & -10 & 7 & 5 & 0 \\
0 & 0 & 0 & 0 & 16 & 68 & 80 & -9 & -16 & 0 \\
0 & 0 & 0 & 0 & -41 & -98 & 9 & -8 & -5 & 0 \\
0 & 0 & 0 & -1 & 18 & -164 & -62 & -42 & 9 & 0 \\
0 & 0 & 0 & 7 & 78 & 212 & -10 & -11 & -3 & 0 \\
0 & 0 & 0 & -19 & -52 & 266 & -34 & 37 & 0 & 0 \\
0 & 0 & 0 & 29 & -158 & -196 & -83 & -8 & 0 & 0 \\
0 & 0 & 0 & -10 & 85 & -377 & 44 & -23 & 0 & 0 \\
0 & 0 & -3 & -47 & 237 & -24 & 48 & 16 & 0 & 0 \\
0 & 0 & 16 & 48 & -24 & 237 & -47 & -3 & 0 & 0 \\
0 & 0 & -23 & 44 & -377 & 85 & -10 & 0 & 0 & 0 \\
0 & 0 & -8 & -83 & -196 & -158 & 29 & 0 & 0 & 0 \\
0 & 0 & 37 & -34 & 266 & -52 & -19 & 0 & 0 & 0 \\
0 & -3 & -11 & -10 & 212 & 78 & 7 & 0 & 0 & 0 \\
0 & 9 & -42 & -62 & -164 & 18 & -1 & 0 & 0 & 0 \\
0 & -5 & -8 & 9 & -98 & -41 & 0 & 0 & 0 & 0 \\
0 & -16 & -9 & 80 & 68 & 16 & 0 & 0 & 0 & 0 \\
0 & 5 & 7 & -10 & 30 & -2 & 0 & 0 & 0 & 0 \\
-1 & 5 & 16 & -34 & -32 & 0 & 0 & 0 & 0 & 0 \\
0 & -6 & -2 & -1 & 9 & 0 & 0 & 0 & 0 & 0 \\
0 & 2 & -11 & 16 & -1 & 0 & 0 & 0 & 0 & 0 \\
0 & 0 & 6 & -7 & 0 & 0 & 0 & 0 & 0 & 0 \\
0 & 0 & -1 & 1 & 0 & 0 & 0 & 0 & 0 & 0
\end{array}\right)
$$

$7_{7}$

$$
\left(\begin{array}{cccccccc}
0 & 0 & 0 & 1 & -1 & 0 & 0 & 0 \\
0 & 0 & 0 & -7 & 8 & 0 & 0 & 0 \\
0 & 0 & 0 & 15 & -19 & 1 & 0 & 0 \\
0 & 0 & 3 & 1 & -4 & -6 & 0 & 0 \\
0 & 0 & -18 & -30 & 59 & 11 & 0 & 0 \\
0 & 0 & 23 & -1 & 0 & -1 & 0 & 0 \\
0 & 3 & 27 & 41 & -123 & -8 & -2 & 0 \\
0 & -11 & -65 & 7 & -2 & 1 & 7 & 0 \\
0 & 4 & -19 & -29 & 130 & -28 & -7 & 0 \\
1 & 20 & 84 & -46 & 35 & 16 & -7 & 0 \\
0 & -7 & 16 & 35 & -46 & 84 & 20 & 1 \\
0 & -7 & -28 & 130 & -29 & -19 & 4 & 0 \\
0 & 7 & 1 & -2 & 7 & -65 & -11 & 0 \\
0 & -2 & -8 & -123 & 41 & 27 & 3 & 0 \\
0 & 0 & -1 & 0 & -1 & 23 & 0 & 0 \\
0 & 0 & 11 & 59 & -30 & -18 & 0 & 0 \\
0 & 0 & -6 & -4 & 1 & 3 & 0 & 0 \\
0 & 0 & 1 & -19 & 15 & 0 & 0 & 0 \\
0 & 0 & 0 & 8 & -7 & 0 & 0 & 0 \\
0 & 0 & 0 & -1 & 1 & 0 & 0 & 0
\end{array}\right)
$$


$8_{1}$

$$
\left(\begin{array}{rrrrrrr}
0 & 1 & -2 & 1 & 0 & 0 & 0 \\
0 & -1 & 6 & -5 & 0 & 0 & 0 \\
0 & 0 & 0 & 6 & -3 & 0 & 0 \\
0 & 0 & -4 & 5 & 10 & 0 & 0 \\
0 & 0 & 0 & 3 & 0 & 3 & 0 \\
0 & 0 & 0 & -10 & -12 & -5 & 0 \\
-1 & -4 & -10 & -20 & -10 & -4 & -1 \\
0 & -5 & -12 & -10 & 0 & 0 & 0 \\
0 & 3 & 0 & 3 & 0 & 0 & 0 \\
0 & 0 & 10 & 5 & -4 & 0 & 0 \\
0 & 0 & -3 & 6 & 0 & 0 & 0 \\
0 & 0 & 0 & -5 & 6 & -1 & 0 \\
0 & 0 & 0 & 1 & -2 & 1 & 0
\end{array}\right)
$$

$$
\left(\begin{array}{rrrrrrrrl}
0 & -1 & 0 & 0 & 0 & 0 & 0 & 0 & 0 \\
0 & 2 & 0 & 0 & 0 & 0 & 0 & 0 & 0 \\
1 & -1 & 0 & 0 & 0 & 0 & 0 & 0 & 0 \\
0 & 0 & -2 & 0 & 0 & 0 & 0 & 0 & 0 \\
0 & 0 & 7 & 0 & 0 & 0 & 0 & 0 & 0 \\
0 & -3 & -8 & 0 & 0 & 0 & 0 & 0 & 0 \\
0 & 7 & -4 & -1 & 0 & 0 & 0 & 0 & 0 \\
0 & 9 & 14 & 5 & 0 & 0 & 0 & 0 & 0 \\
0 & -5 & -1 & -8 & 0 & 0 & 0 & 0 & 0 \\
0 & 0 & -28 & 1 & 0 & 0 & 0 & 0 & 0 \\
0 & 0 & 17 & -2 & 0 & 0 & 0 & 0 & 0 \\
0 & 0 & 56 & 29 & 0 & 0 & 0 & 0 & 0 \\
0 & 0 & -1 & -17 & 5 & 0 & 0 & 0 & 0 \\
0 & 0 & -32 & -77 & -24 & 0 & 0 & 0 & 0 \\
0 & 0 & 10 & 33 & 26 & 0 & 0 & 0 & 0 \\
0 & 0 & 0 & 143 & 36 & 0 & 0 & 0 & 0 \\
0 & 0 & 0 & 29 & -43 & 0 & 0 & 0 & 0 \\
0 & 0 & 0 & -87 & -108 & 0 & 0 & 0 & 0 \\
0 & 0 & 0 & -24 & 47 & -10 & 0 & 0 & 0 \\
0 & 0 & 0 & 42 & 192 & 42 & 0 & 0 & 0 \\
0 & 0 & 0 & -10 & 47 & -24 & 0 & 0 & 0 \\
0 & 0 & 0 & 0 & -108 & -87 & 0 & 0 & 0 \\
0 & 0 & 0 & 0 & -43 & 29 & 0 & 0 & 0 \\
0 & 0 & 0 & 0 & 36 & 143 & 0 & 0 & 0 \\
0 & 0 & 0 & 0 & 26 & 33 & 10 & 0 & 0 \\
0 & 0 & 0 & 0 & -24 & -77 & -32 & 0 & 0 \\
0 & 0 & 0 & 0 & 5 & -17 & -1 & 0 & 0 \\
0 & 0 & 0 & 0 & 0 & 29 & 56 & 0 & 0 \\
0 & 0 & 0 & 0 & 0 & -2 & 17 & 0 & 0 \\
0 & 0 & 0 & 0 & 0 & 1 & -28 & 0 & 0 \\
0 & 0 & 0 & 0 & 0 & -8 & -1 & -5 & 0 \\
0 & 0 & 0 & 0 & 0 & 5 & 14 & 9 & 0 \\
0 & 0 & 0 & 0 & 0 & -1 & -4 & 7 & 0 \\
0 & 0 & 0 & 0 & 0 & 0 & -8 & -3 & 0 \\
0 & 0 & 0 & 0 & 0 & 0 & 7 & 0 & 0 \\
0 & 0 & 0 & 0 & 0 & 0 & -2 & 0 & 0 \\
0 & 0 & 0 & 0 & 0 & 0 & 0 & -1 & 1 \\
0 & 0 & 0 & 0 & 0 & 0 & 0 & 2 & 0 \\
0 & 0 & 0 & 0 & 0 & 0 & 0 & -1 & 0 \\
0 & & & & & & & \\
0 & & 0
\end{array}\right)
$$




$\mathbf{8}_{5} \quad\left(\begin{array}{rrrrrrrrrr}0 & -1 & 0 & 0 & 0 & 0 & 0 & 0 & 0 & 0 \\ 0 & 3 & -1 & 0 & 0 & 0 & 0 & 0 & 0 & 0 \\ 1 & -7 & 4 & 0 & 0 & 0 & 0 & 0 & 0 & 0 \\ 0 & 8 & -10 & 0 & 0 & 0 & 0 & 0 & 0 & 0 \\ 0 & 3 & 12 & 0 & 0 & 0 & 0 & 0 & 0 & 0 \\ 0 & -12 & 0 & 1 & 0 & 0 & 0 & 0 & 0 & 0 \\ 0 & 11 & -15 & -6 & 0 & 0 & 0 & 0 & 0 & 0 \\ 0 & 7 & -3 & 20 & 0 & 0 & 0 & 0 & 0 & 0 \\ 0 & -5 & 40 & -43 & 0 & 0 & 0 & 0 & 0 & 0 \\ 0 & 0 & -19 & 28 & 0 & 0 & 0 & 0 & 0 & 0 \\ 0 & 0 & -27 & 42 & 1 & 0 & 0 & 0 & 0 & 0 \\ 0 & 0 & 68 & -66 & -11 & 0 & 0 & 0 & 0 & 0 \\ 0 & 0 & -10 & -15 & 41 & 0 & 0 & 0 & 0 & 0 \\ 0 & 0 & -29 & 93 & -66 & 0 & 0 & 0 & 0 & 0 \\ 0 & 0 & 10 & -16 & 23 & 0 & 0 & 0 & 0 & 0 \\ 0 & 0 & 0 & -37 & 61 & 0 & 0 & 0 & 0 & 0 \\ 0 & 0 & 0 & 104 & -107 & -5 & 0 & 0 & 0 & 0 \\ 0 & 0 & 0 & -71 & 64 & 34 & 0 & 0 & 0 & 0 \\ 0 & 0 & 0 & -42 & 127 & -76 & 0 & 0 & 0 & 0 \\ 0 & 0 & 0 & 46 & -95 & 32 & 0 & 0 & 0 & 0 \\ 0 & 0 & 0 & -10 & -70 & 96 & 0 & 0 & 0 & 0 \\ 0 & 0 & 0 & 0 & 127 & -127 & 0 & 0 & 0 & 0 \\ 0 & 0 & 0 & 0 & -96 & 70 & 10 & 0 & 0 & 0 \\ 0 & 0 & 0 & 0 & -32 & 95 & -46 & 0 & 0 & 0 \\ 0 & 0 & 0 & 0 & 76 & -127 & 42 & 0 & 0 & 0 \\ 0 & 0 & 0 & 0 & -34 & -64 & 71 & 0 & 0 & 0 \\ 0 & 0 & 0 & 0 & 5 & 107 & -104 & 0 & 0 & 0 \\ 0 & 0 & 0 & 0 & 0 & -61 & 37 & 0 & 0 & 0 \\ 0 & 0 & 0 & 0 & 0 & -23 & 16 & -10 & 0 & 0 \\ 0 & 0 & 0 & 0 & 0 & 66 & -93 & 29 & 0 & 0 \\ 0 & 0 & 0 & 0 & 0 & -41 & 15 & 10 & 0 & 0 \\ 0 & 0 & 0 & 0 & 0 & 11 & 66 & -68 & 0 & 0 \\ 0 & 0 & 0 & 0 & 0 & -1 & -42 & 27 & 0 & 0 \\ 0 & 0 & 0 & 0 & 0 & 0 & -28 & 19 & 0 & 0 \\ 0 & 0 & 0 & 0 & 0 & 0 & 43 & -40 & 5 & 0 \\ 0 & 0 & 0 & 0 & 0 & 0 & -20 & 3 & -7 & 0 \\ 0 & 0 & 0 & 0 & 0 & 0 & 6 & 15 & -11 & 0 \\ 0 & 0 & 0 & 0 & 0 & 0 & -1 & 0 & 12 & 0 \\ 0 & 0 & 0 & 0 & 0 & 0 & 0 & -12 & -3 & 0 \\ 0 & 0 & 0 & 0 & 0 & 0 & 0 & 10 & -8 & 0 \\ 0 & 0 & 0 & 0 & 0 & 0 & 0 & -4 & 7 & -1 \\ 0 & 0 & 0 & 0 & 0 & 0 & 0 & 1 & -3 & 0 \\ 0 & 0 & 0 & 0 & 0 & 0 & 0 & 0 & 1 & 0 \\ 0 & & & & & & & & \\ 0 & \end{array}\right)$


$8_{20}$

$$
\left(\begin{array}{rrrrrr}
0 & 0 & 0 & 1 & -1 & 0 \\
0 & 0 & 0 & -5 & 1 & 0 \\
0 & 0 & -2 & 0 & -2 & 0 \\
0 & -1 & -4 & 3 & 2 & 0 \\
-1 & -5 & 0 & -3 & 1 & 0 \\
0 & 1 & -3 & 0 & -5 & -1 \\
0 & 2 & 3 & -4 & -1 & 0 \\
0 & -2 & 0 & -2 & 0 & 0 \\
0 & 1 & -5 & 0 & 0 & 0 \\
0 & -1 & 1 & 0 & 0 & 0
\end{array}\right)
$$

$9_{1}$

$$
\left(\begin{array}{ll}
1 & 0 \\
0 & 0 \\
0 & 0 \\
0 & 0 \\
0 & 0 \\
0 & 0 \\
0 & 0 \\
0 & 0 \\
0 & 0 \\
0 & 1
\end{array}\right)
$$

$$
\left(\begin{array}{rrrrcrrr}
1 & -3 & 3 & -1 & 0 & 0 & 0 & 0 \\
0 & 6 & -12 & 6 & 0 & 0 & 0 & 0 \\
0 & 4 & 5 & -12 & 3 & 0 & 0 & 0 \\
0 & 0 & 15 & 2 & -13 & 0 & 0 & 0 \\
0 & 0 & 10 & 5 & 9 & -3 & 0 & 0 \\
0 & 0 & 0 & 15 & 16 & 8 & 0 & 0 \\
0 & 0 & 0 & 20 & 0 & 0 & 1 & 0 \\
0 & 0 & 0 & 0 & -15 & -5 & -1 & 0 \\
0 & -1 & -5 & -15 & 0 & 0 & 0 & 0 \\
0 & 1 & 0 & 0 & 20 & 0 & 0 & 0 \\
0 & 0 & 8 & 16 & 15 & 0 & 0 & 0 \\
0 & 0 & -3 & 9 & 5 & 10 & 0 & 0 \\
0 & 0 & 0 & -13 & 2 & 15 & 0 & 0 \\
0 & 0 & 0 & 3 & -12 & 5 & 4 & 0 \\
0 & 0 & 0 & 0 & 6 & -12 & 6 & 0 \\
0 & 0 & 0 & 0 & -1 & 3 & -3 & 1
\end{array}\right)
$$




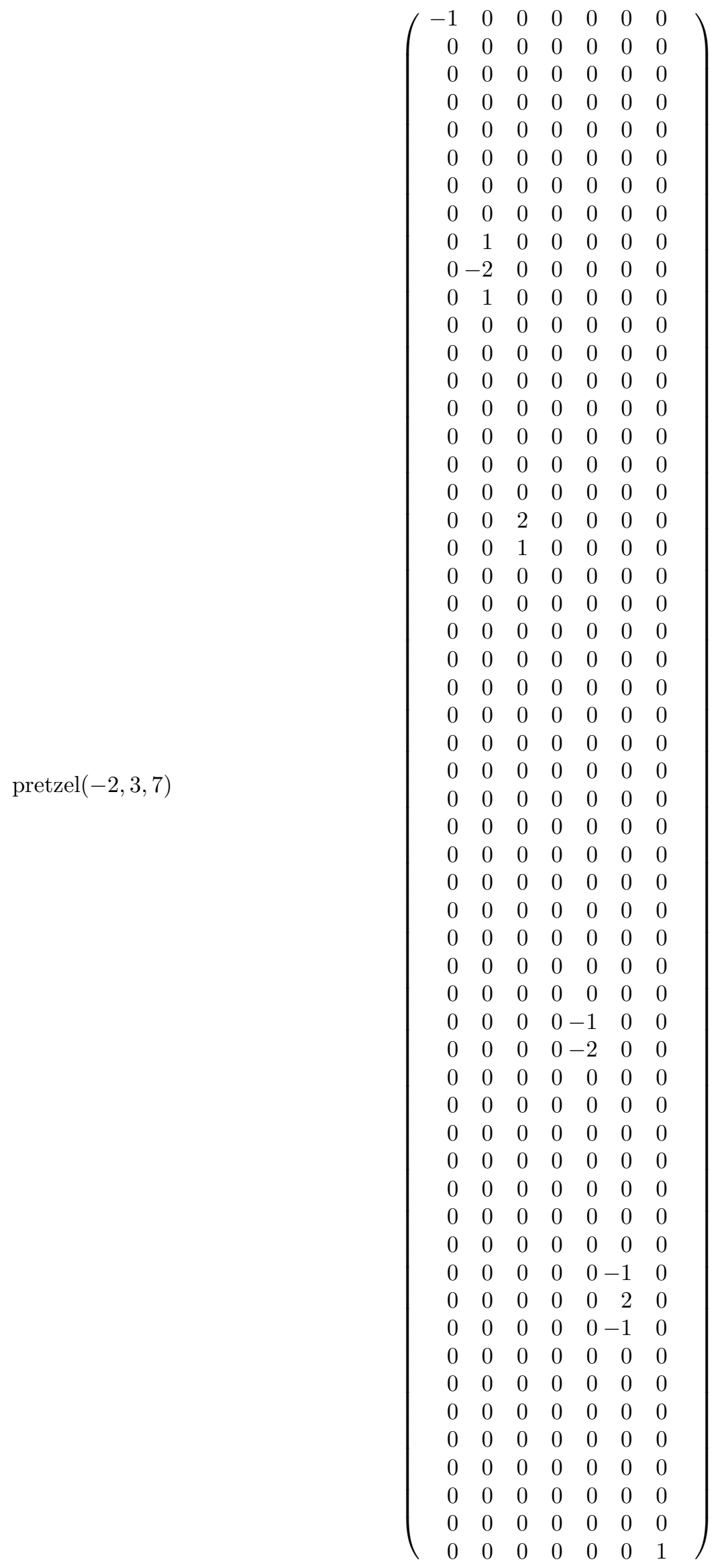

